\title{
A generalized vortex ring model
}

\section{FELIX KAPLANSKI ${ }^{1}$, SERGEI S SAZHIN ${ }^{2} \dagger$, YASUHIDE FUKUMOTO ${ }^{3}$, STEVEN BEGG AND MORGAN HEIKAL ${ }^{2}$}

\author{
${ }^{1}$ Laboratory of Multiphase Physics, Tallinn University of Technology, Tallinn 19086, Estonia \\ ${ }^{2}$ School of Environment and Technology, Faculty of Science and Engineering, The University of \\ Brighton, Brighton BN2 4GJ, UK \\ ${ }^{3}$ Graduate School of Mathematics and Mathematical Research Centre for Industrial Technology, \\ Kyushu University 33, Fukuoka 812-8581, Japan
}

(Received 29 October 2007 and in revised form 28 October 2008)

A conventional laminar vortex ring model is generalized by assuming that the time dependence of the vortex ring thickness $\ell$ is given by the relation $\ell=a t^{b}$, where $a$ is a positive number and $1 / 4 \leqslant b \leqslant 1 / 2$. In the case in which $a=\sqrt{2 v}$, where $v$ is the laminar kinematic viscosity, and $b=1 / 2$, the predictions of the generalized model are identical with the predictions of the conventional laminar model. In the case of $b=1 / 4$ some of its predictions are similar to the turbulent vortex ring models, assuming that the time-dependent effective turbulent viscosity $v_{*}$ is equal to $\ell \ell^{\prime}$. This generalization is performed both in the case of a fixed vortex ring radius $R_{0}$ and increasing vortex ring radius. In the latter case, the so-called second Saffman's formula is modified. In the case of fixed $R_{0}$, the predicted vorticity distribution for short times shows a close agreement with a Gaussian form for all $b$ and compares favourably with available experimental data. The time evolution of the location of the region of maximal vorticity and the region in which the velocity of the fluid in the frame of reference moving with the vortex ring centroid is equal to zero is analysed. It is noted that the locations of both regions depend upon $b$, the latter region being always further away from the vortex axis than the first one. It is shown that the axial velocities of the fluid in the first region are always greater than the axial velocities in the second region. Both velocities depend strongly upon $b$. Although the radial component of velocity in both of these regions is equal to zero, the location of both of these regions changes with time. This leads to the introduction of an effective radial velocity component; the latter case depends upon $b$. The predictions of the model are compared with the results of experimental measurements of vortex ring parameters reported in the literature.

\section{Introduction}

Vortex rings have been widely observed as persistent slowly decaying structures (Saffman 1992). These structures provide a relatively simple flow field, accessible to experimental, numerical and theoretical studies. The importance of vortex rings was emphasized by Saffman (1992) who wrote, 'This commonly known phenomenon exemplifies the whole range of problems of vortex motion'. The properties of the vortex rings have been studied for over a century both theoretically and experimentally 
(Helmholtz 1858; Lamb 1932; Phillips 1956; Norbury 1973; Kambe \& Oshima 1975; Saffman 1992; Shariff \& Leonard 1992; Lim \& Nickels 1995). Recent developments on the modelling side include Stanaway, Cantwell \& Spalart (1988), Rott \& Cantwell (1993a,b), Mohseni \& Gharib (1998), Kaplanski \& Rudi (1999, 2005), Fukumoto \& Moffatt (2000), Shusser \& Gharib (2000), Mohseni (2001, 2006), Linden \& Turner (2001) and Fukumoto \& Kaplanski (2008).

Classical vortex rings are generated by a moving piston, pushing a liquid column of length $L$ through an orifice or nozzle of diameter $D$. The flow separates at the edge of the orifice, and a cylindrical vortex sheet forms and rolls up into a vortex ring structure. This structure can be laminar or turbulent depending upon the method of generation and the ambient conditions. Also, the mushroomlike patterns resembling classical vortex ring motion are often observed in nature and industry, including gasoline engines. Such structures can be formed in a fluid when localized forces are applied to the fluid during a short period of time (e.g. in the injection of gasoline in modern engines). In this case, jets with vortex ring structures at the spray periphery can be produced. There is 'some' similarity between the mathematical tools used to describe these structures and those of classical vortex rings.

Saffman (1970) derived an explicit formula for the translational velocity (axial velocity of the centroid as discussed later) of thin-cored laminar vortex rings of radius $R_{0}$ in the form

$$
V_{x}=\frac{\Gamma_{0}}{4 \pi R_{0}}\left[\ln \left(\frac{8 R_{0}}{\sqrt{4 v t}}\right)-0.558+O\left(\frac{\sqrt{v t}}{R_{0}} \ln \left(\frac{v t}{R_{0}^{2}}\right)\right)\right],
$$

where $v$ is the fluid kinematic viscosity and $\Gamma_{0}$ is the initial circulation of the vortex ring, which is conserved. The vorticity distribution inside this ring corresponds to the Lamb-Oseen vortex filament (Lamb 1932). This formula is valid at the initial stage of the vortex ring development.

The description of the final stage of the laminar viscous vortex ring decay can be based on Phillips's (1956) self-similar solution for vorticity distribution and the corresponding stream function. In this case Rott \& Cantwell (1993a) showed that in the limit of large times the translational velocity of vortex rings can be described by the following equation:

$$
V_{x}=\frac{M}{4 \pi^{2} R_{0}^{3}}\left[\frac{7 \sqrt{\pi} R_{0}^{3}}{30(2 v t)^{3 / 2}}\right],
$$

where $M$ is the momentum of vorticity per unit density.

An approximate, linear first-order solution of the Navier-Stokes equation for the axisymmetric geometry and arbitrary time was reported by Kaltaev (1982), Berezovski \& Kaplanski (1995) and Kaplanski \& Rudi (1999). Based on this solution, Kaplanski $\&$ Rudi (2005) derived an expression for the translational velocity of the vortex ring for arbitrary times. In the limit of small and large times this expression reduces to those described by (1.1) and (1.2) respectively (Kaplanski \& Rudi 1999; Fukumoto $\&$ Kaplanski 2008). For the initial stage of vortex ring development, its predictions show good agreement with the results of numerical simulations reported by Stanaway et al. (1988) for Reynolds numbers (defined by the ratio of the circulation to the kinematic viscosity) up to 400 (Fukumoto \& Kaplanski 2008). The effects of these numbers upon the numerical results was shown to be minimal. 
The main limitation of the models mentioned above is that they are based upon the assumption that the vortex ring radius $R_{0}$ remains constant. Saffman (1970) attempted to relax this assumption and, using simple dimensional analysis, derived an alternative formula for $V_{x}$ of the form

$$
V_{x}=\frac{M}{k\left(R_{0}+k^{\prime} v t\right)^{3 / 2}},
$$

where $k$ and $k^{\prime}$ are fitting constants. Weigand \& Gharib (1997) have shown that an appropriate choice of these constants leads to a close match to (1.3) with their original experimental data and the results of rigorous numerical analysis by Stanaway et al. (1988). Both experimental data reported by Weigand \& Gharib (1997) and the model by Kaplanski \& Rudi (2005) predict the Gaussian distribution of the vorticity in the vortex ring. Also, it was shown that the formulae obtained in the limit of small vortex ring Reynolds numbers can be applicable for the description of vortex rings with realistic values of these numbers (see Fukumoto \& Kaplanski 2008).

In contrast to the aforementioned laminar vortex ring models, the theory of turbulent vortex rings is far less developed. To the best of the authors' knowledge, the first attempt to investigate turbulent vortex ring flow structures was made by Lugovtsov (1970) who based his analysis on the introduction of the time-dependent, turbulent (eddy) viscosity

$$
\nu_{*} \propto \ell \ell^{\prime},
$$

where $\ell$ is the diffusivity scale of the ring core (cf. Lavrentiev \& Shabat 1973; Kovasznay, Fujita $\&$ Lee 1974). In our paper we assume that $\ell=\sqrt{2 v t}$ in the case of laminar vortex rings. This definition of $\ell$ is different from the definitions used by some other authors. For example, Saffman (1970) defined $\ell=\sqrt{4 v t}$. Equation (1.4) made it possible to describe vortex rings as self-similar structures. However, the comparison of this model with experimental observations proved inconclusive (Maxworthy 1972, 1974, 1977; Glezer \& Coles 1990; Sazhin et al. 2001; Cantwell 2002). Using (1.4), Lugovtsov $(1970,1976)$ developed a turbulent vortex ring model with turbulent viscosity $v_{*}$ and $\ell \propto t^{1 / 4}$. Further support of this model was provided by Sazhin et al. (2001) who applied it to modelling of turbulent vortex ring structures observed in gasoline engines. At the same time the model suggested by Lugovtsov $(1970,1976)$ was based upon a number of restrictive assumptions, the applicability of which to realistic physical conditions was not evident. The link between this model and the models described by Kaplanski \& Rudi $(1999,2005)$ was not clear. The integral properties of the turbulent vortex rings, such as circulation, kinetic energy and translational velocity, were not derived. As a result, the applicability of the model to realistic physical conditions was not at first evident. This was therefore the main driving force behind this paper in which an attempt is made to generalize the laminar and turbulent vortex ring models by assuming that $\ell \propto t^{b}$, where $1 / 4 \leqslant b \leqslant 1 / 2$. This model is expected to incorporate both the laminar and turbulent vortex ring models described earlier for the limiting values of $b$.

The basic equations and approximations of the new model are described in $\S 2$. The analytical solutions of the equations, describing this model, are presented and discussed in $\S 3$. In $\S 4$ the limiting cases of the solutions of these equations for long and short times are discussed. The solutions are validated against experimental data, available in the literature, where possible. The results are presented in $\S 5$. The main results of the paper are summarized in $\S 6$. 


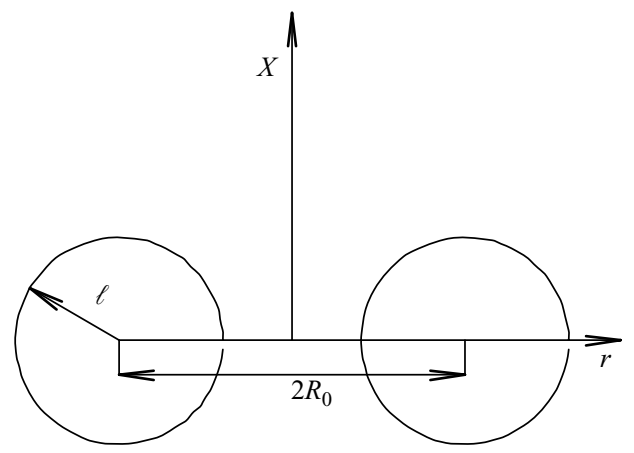

FIGURE 1. A schematic presentation of the vortex ring with $\ell=a t^{b}$.

\section{Basic equations and approximations}

The general vorticity equation for incompressible flows follows from the NavierStokes equation and can be presented in the form (e.g. Panton 1996)

$$
\frac{D \zeta}{D t}=\zeta \cdot \nabla v+v \nabla^{2} \zeta
$$

where $\zeta=\nabla \times v$ is the vorticity, $v$ is the kinematic viscosity.

Assuming that the flow is axisymmetric, when the vector $\zeta$ has only one azimuthal component $\zeta,(2.1)$ can be simplified to (Batchelor 1967)

$$
\frac{\partial \zeta}{\partial t}+\frac{\partial\left(v_{x} \zeta\right)}{\partial x}+\frac{\partial\left(v_{r} \zeta\right)}{\partial r}=v\left[\frac{\partial^{2} \zeta}{\partial x^{2}}+\frac{\partial^{2} \zeta}{\partial r^{2}}+\frac{1}{r} \frac{\partial \zeta}{\partial r}-\frac{\zeta}{r^{2}}\right]
$$

where the meaning of $r$ and $x$ axes is shown in figure 1.

The stream function $\Psi$ is introduced as

$$
v_{x}=\frac{1}{r} \frac{\partial \Psi}{\partial r}+V_{x}, \quad v_{r}=-\frac{1}{r} \frac{\partial \Psi}{\partial x},
$$

where $V_{x}$ is the velocity of the centroid at $r=0$ and

$$
x=x_{0}=\frac{\int_{0}^{\infty} \int_{-\infty}^{\infty} 2 \pi r x \zeta \mathrm{d} x \mathrm{~d} r}{\int_{0}^{\infty} \int_{-\infty}^{\infty} 2 \pi r \zeta \mathrm{d} x \mathrm{~d} r}
$$

$V_{x}=\mathrm{d} x_{0}(t) / \mathrm{d} t$. From the definition of $\zeta$ follows the equation

$$
\frac{\partial^{2} \Psi}{\partial x^{2}}+\frac{\partial^{2} \Psi}{\partial r^{2}}-\frac{1}{r} \frac{\partial \Psi}{\partial r}=-r \zeta
$$

Equations (2.2)-(2.4) describe any axisymmetric flow, including vortex rings. In the latter case, physically meaningful solutions of these equations should satisfy the following boundary conditions: both $\zeta$ and $\Psi$ are equal to zero at $r=0$ and approach zero when $\sqrt{x^{2}+r^{2}} \rightarrow \infty$.

Following Berezovski \& Kaplanski (1995), we introduce the following dimensionless variables and parameters:

$$
\sigma=\frac{r}{\ell}, \quad \eta=\frac{x-x_{0}(t)}{\ell}, \quad \theta=\frac{R_{0}}{\ell}, \quad \Phi=\frac{\Psi}{\zeta_{0} \ell^{3}}, \quad \omega=\frac{\zeta}{\zeta_{0}}, \quad \zeta_{0}=A t^{-\lambda},
$$

where $R_{0}$ is the free parameter of the model which is usually identified with the initial radius of the vortex ring (the value of $r$ at which the axial velocity in the frame of reference moving with $V_{x}$ reaches its local minimum at $\eta=0$ ); the length $\ell$ 
can be identified with the thickness of the vortex ring as shown in figure 1; and the parameter $A$ can be identified with the initial vorticity at an a priori chosen location. As mentioned in $\S 1$, in the case of a laminar vortex ring it was assumed that $\ell=\sqrt{2 v t}$ (Berezovski \& Kaplanski 1995). In our case, a more general assumption is made, such that

$$
\ell=a t^{b} .
$$

In the case in which $a=\sqrt{2 v}$ and $b=1 / 2$, the value of $\ell$ defined by (2.5) reduces to the one considered by Berezovski \& Kaplanski (1995). In the case of $b=1 / 4$ and the long time limit, the model essentially reduces to the one described by Lugovtsov (1976) for the turbulent rings. One can expect that for real-life vortex-ring-like structures, the values of $b$ lie in the range $1 / 4 \leqslant b \leqslant 1 / 2$. Hence, the analysis of the model will focus on this range of $b$.

Also, we will assume that $v$ is not constant but changes with time (although it remains homogeneous in space). For this case we can formally replace $v$ in (2.2) with an effective viscosity $\nu_{*}$. It is expected that this generalization of the vortex ring model can incorporate the effects of turbulence.

The assumption of spatially homogeneous but time-dependent effective viscosity $\nu_{*}$ is similar to that made by Lugovtsov (1976) for turbulent viscosity. In practice this viscosity is expected to decrease from its maximal value near the maximal vorticity region to zero at long distances from the vortex ring. This effect, however, is not important for the analysis of the vortex ring dynamics, since the most important effect of viscosity comes from the region in which it is maximal (Lugovtsov 1976).

Remembering the definitions of the above-mentioned dimensionless variables and parameters and $\ell$ and replacing $v$ by $\nu_{*}$ in (2.2), the equation can be rewritten in the following form:

$$
\begin{array}{r}
-\frac{b \ell^{2}}{\mathcal{V}_{*} t}\left[\frac{\lambda}{b} \omega+\theta \frac{\partial \omega}{\partial \theta}+\sigma \frac{\partial \omega}{\partial \sigma}+\eta \frac{\partial \omega}{\partial \eta}\right]+R e[ \\
\left.-\frac{\partial}{\partial \sigma}\left[\frac{\omega}{\sigma} \frac{\partial \Phi}{\partial \eta}\right]+\frac{\partial}{\partial \eta}\left[\frac{\omega}{\sigma} \frac{\partial \Phi}{\partial \sigma}\right]\right] \\
=\frac{\partial^{2} \omega}{\partial \sigma^{2}}+\frac{\partial^{2} \omega}{\partial \eta^{2}}+\frac{1}{\sigma} \frac{\partial \omega}{\partial \sigma}-\frac{\omega}{\sigma^{2}}
\end{array}
$$

where the vortex ring Reynolds number is defined as $R e=\zeta_{0} \ell^{2} / \nu_{*}$.

It should be noted that $R e$ introduced in our paper is time-dependent.

Further development of this model requires the specification of $v_{*}(t)$. Following Lugovtsov (1976), one can make a formal dimensionally correct assumption that $\nu_{*}=\ell \ell^{\prime}$, where $\ell^{\prime}=\mathrm{d} \ell / \mathrm{d} t$ (cf. $\S 1$ ). Remembering (2.5), this assumption leads to the following relation:

$$
\nu_{*}=\ell \ell^{\prime}=a^{2} b t^{2 b-1} .
$$

In most realistic physical conditions we expect that the viscosity does not increase with time and that the thickness of the vortex ring does not decrease with time. This imposes the following restriction on the values of $b$ :

$$
0 \leqslant b \leqslant 1 / 2 \text {. }
$$

As shown later (see (3.3)), the vortex ring Reynolds number is conserved for $b=1 / 4$, decreases with time for $1 / 4<b \leqslant 1 / 2$ and increases with time for $0 \leqslant b<1 / 4$. The latter process has no physical grounds, and the condition (2.8) is restricted to

$$
1 / 4 \leqslant b \leqslant 1 / 2 .
$$


The values of $b$ in the range (2.9) and $a$ can be considered free parameters. Their values will be estimated based upon the comparison of the predictions of the model with experimental data.

Using (2.5) and (2.7), it can be shown that

$$
\frac{b \ell^{2}}{v_{*} t}=1 \text {. }
$$

Using this result, one can see that for $b=1 / 2,(2.6)$ is identical to (8) of Kaplanski \& Rudi (1999) if one remembers that $a^{2} b=v$ when $a=\sqrt{2 v}$.

To simplify (2.6) further, the term proportional to $R e$ can be rewritten as

$$
\mathscr{R} \equiv \operatorname{Re}\left[-\frac{\partial}{\partial \sigma}\left[\frac{\omega}{\sigma} \frac{\partial}{\partial \eta}\right]+\frac{\partial}{\partial \eta}\left[\frac{\omega}{\sigma} \frac{\partial}{\partial \sigma}\right]\right] \Psi=\frac{R e}{\ell^{2}}\left[\frac{\omega}{\sigma} \operatorname{div}(v)+v \nabla \frac{\omega}{\sigma}\right] .
$$

At the initial stage of vortex ring development, the core is thin; the streamlines are practically circular; and fluid velocities are almost perpendicular to the gradient of vorticity. This allows one to assume that the term proportional to $v \nabla(\omega / \sigma)$ is small at this point. Since $\operatorname{div}(\boldsymbol{v})=0$ for incompressible flows, one can ignore the contribution of $\mathscr{R}$ in (2.10) in this case. In the final stage of vortex ring development, the contribution of this term can be ignored, as $R e$ approaches zero. The estimate of $\mathscr{R}$ in the intermediate stage is more difficult to determine. Assuming that the term proportional to $\operatorname{Re}(\mathscr{R})$ is close to zero, (2.6) is simplified to

$$
-\frac{\lambda}{b} \omega-\theta \frac{\partial \omega}{\partial \theta}-\sigma \frac{\partial \omega}{\partial \sigma}-\eta \frac{\partial \omega}{\partial \eta}=\frac{\partial^{2} \omega}{\partial \sigma^{2}}+\frac{\partial^{2} \omega}{\partial \eta^{2}}+\frac{1}{\sigma} \frac{\partial \omega}{\partial \sigma}-\frac{\omega}{\sigma^{2}} .
$$

The range of applicability of (2.11) will be investigated more rigorously later based on the comparison of its predictions with available experimental data for non-zero values of $R e$.

Although the values of $\omega$ predicted by (2.11) vary in time and space, the specific momentum of the vortex ring defined by the expression

$$
M=\pi \int_{0}^{\infty} \int_{-\infty}^{\infty} r^{2} \zeta \mathrm{d} x \mathrm{~d} r
$$

is conserved even in the turbulent case (Lugovtsov 1976).

\section{Analytical solutions}

The linearized form of (2.2) (dimensional form of (2.11)) was solved subject to the initial condition (Fukumoto \& Kaplanski 2008)

$$
\zeta_{0}=\Gamma_{0} \delta(x) \delta\left(r-R_{0}\right)
$$

where $\Gamma_{0}$ is the initial circulation. The dimensionless form of this solution for $\lambda=4 b$ can be presented as

$$
\omega=\frac{\sigma}{2} \exp \left[-\frac{1}{2}\left(\sigma^{2}+\eta^{2}+\theta^{2}\right)\right]\left[I_{0}(\sigma \theta)-I_{2}(\sigma \theta)\right],
$$

where $I_{0}$ and $I_{2}$ are modified Bessel functions.

Note that (3.1) coincides with the solution of the original system of equations (2.2)-(2.4) subject to the same initial condition, valid for arbitrary $R e$, in the limit of short and long times. This is an expected result, since for long times $R e \rightarrow 0$, and for short times the multiple of $R e$ in (2.6) tends to zero, as follows from the earlier 
presented qualitative analysis. Note that in the limit of short times, (3.1) reduces to the Oseen solution for the decaying line vortex (see Panton 1996).

From the conservation of $M$ (see (2.12)) it follows that

$$
\zeta_{0}=\frac{M}{\pi \sqrt{2 \pi}} a^{-4} t^{-4 b}=\frac{M}{\pi \sqrt{2 \pi}} a^{-4} t^{-\lambda}
$$

This yields

$$
R e=\frac{\zeta_{0} \ell^{2}}{\nu_{*}}=\frac{M}{\pi \sqrt{2 \pi} b} a^{-4} t^{1-4 b}=\frac{M}{\pi \sqrt{2 \pi} b} a^{-4} t^{1-\lambda} .
$$

As follows from (3.3), the Reynolds number is conserved for $b=1 / 4$. This property of $R e$ has turned out to be convenient for the analysis of developed turbulent vortex ring flows (Cantwell 2002). For $1 / 4<b \leqslant 1 / 2$ (cf. condition (2.9)) the Reynolds number decreases with time following the power law. For $0<b<1 / 4, R e$ would increase with time. This is not consistent with the physical background of the phenomenon.

Although solution (3.1) was derived based upon the assumption that the nonlinear terms proportional to $R e$ in (2.6) are negligible, it is thought that it can be applied to the analysis of real-life laminar and turbulent vortex ring flows (see $\S 2$ ).

Remembering that

$$
I_{1}(x)=\frac{x\left(I_{0}(x)-I_{2}(x)\right)}{2}
$$

(3.1) can be rewritten as

$$
\tilde{\omega}=\exp \left[-\frac{1}{2}\left(\sigma^{2}+\eta^{2}+\theta^{2}\right)\right] I_{1}(\sigma \theta)
$$

where

$$
\begin{aligned}
\tilde{\omega} & =\zeta / \tilde{\zeta}_{0}, \\
\tilde{\zeta}_{0} & =\zeta_{0} / \theta=\frac{M}{\pi \sqrt{2 \pi} R_{0}} a^{-3} t^{-3 b}=\frac{M}{\pi \sqrt{2 \pi} R_{0}} a^{-3} t^{-\lambda} .
\end{aligned}
$$

Note that $\lambda$ in this case is equal to $3 b$, due to the different choice of the normalizing parameter $\tilde{\zeta}_{0}$. Equation (3.5) is identical to the one used by Kaplanski \& Rudi (1999).

Following earlier approaches to the analysis of this problem (see Kaplanski \& Rudi 1999, 2005), the focus is directed to (3.5) and (3.6). The tilde ( ) will be omitted to simplify the notation. Note, dimensional forms of the solutions of (3.1) and (3.5) are identical.

Once the value of vorticity has been found, the dimensionless stream function $\Phi$ can be calculated in exactly the same manner as Kaplanski \& Rudi (1999). This is given by the following equation, which follows from (2.4):

$$
\Phi=\frac{M \sigma}{4 \pi R_{0} \zeta_{0} \ell^{3}} \int_{0}^{\infty} F(\mu, \eta) J_{1}(\theta \mu) J_{1}(\sigma \mu) \mathrm{d} \mu=\frac{\sigma \sqrt{2 \pi}}{4} \int_{0}^{\infty} F(\mu, \eta) J_{1}(\theta \mu) J_{1}(\sigma \mu) \mathrm{d} \mu,
$$

where

$$
\begin{aligned}
& F(\mu, \eta)=\exp (\eta \mu) \operatorname{erfc}\left(\frac{\mu+\eta}{\sqrt{2}}\right)+\exp (-\eta \mu) \operatorname{erfc}\left(\frac{\mu-\eta}{\sqrt{2}}\right), \\
& \operatorname{erfc}(x)=\frac{2}{\sqrt{\pi}} \int_{x}^{\infty} \exp \left(-t^{2}\right) \mathrm{d} t=1-\operatorname{erf}(x)=1-\frac{2}{\sqrt{\pi}} \int_{0}^{x} \exp \left(-t^{2}\right) \mathrm{d} t
\end{aligned}
$$

and $J_{0}$ and $J_{1}$ are Bessel functions; when deriving (3.7), (3.5) was taken into account. 
Once the value of $\Phi$ has been found, the components of velocity can be calculated from (2.3). At this stage, the dimensionless velocities need to be defined. Following Saffman (1970), our analysis is based upon the following normalizations: $u_{x} \equiv$ $\left(v_{x}-V_{x}\right) / v_{n}$ and $u_{r} \equiv v_{r} / v_{n}$, where

$$
v_{n}=\frac{M}{4 \pi^{2} R_{0}^{3}}=\frac{\Gamma_{0}}{4 \pi R_{0}} ;
$$

$\Gamma_{0}=M /\left(\pi R_{0}^{2}\right)$ is the initial circulation of the vortex ring (Kaplanski \& Rudi 2005). Remembering this definition of $v_{n}$ and (2.3) and (3.7), the following expressions have been obtained:

$$
\begin{gathered}
u_{x}=\pi \theta^{2} \int_{0}^{\infty} \mu F(\mu, \eta) J_{1}(\theta \mu) J_{0}(\sigma \mu) \mathrm{d} \mu, \\
u_{r}=-\pi \theta^{2} \int_{0}^{\infty} \mu \tilde{F}(\mu, \eta) J_{1}(\theta \mu) J_{1}(\sigma \mu) \mathrm{d} \mu,
\end{gathered}
$$

where

$$
\tilde{F}(\mu, \eta)=\exp (\eta \mu) \operatorname{erfc}\left(\frac{\mu+\eta}{\sqrt{2}}\right)-\exp (-\eta \mu) \operatorname{erfc}\left(\frac{\mu-\eta}{\sqrt{2}}\right) .
$$

Once the values of the vorticity and stream function for the vortex ring have been obtained, then the dimensional energy $E$ of the vortex ring can be calculated using the following equation (Batchelor 1967):

$$
E=\pi \rho \int_{0}^{\infty} \mathrm{d} r \int_{-\infty}^{+\infty} \zeta \Psi \mathrm{d} x
$$

As in the case of velocities, there are several ways to normalize $E$. In our analysis, following Saffman (1992), $E$ will be normalized by $E_{0}=\rho \Gamma_{0}^{2} R_{0} / 2=\rho M^{2} /\left(2 \pi^{2} R_{0}^{3}\right)$.

Using (2.4), (3.5) and (3.10), we obtain, following the approach developed by Kaplanski \& Rudi (2005),

$$
\tilde{E}=\frac{E}{E_{0}}=\frac{\sqrt{\pi} \theta^{3}}{12}{ }_{2} F_{2}\left[\frac{3}{2}, \frac{3}{2} ; \frac{5}{2}, 3 ;-\theta^{2}\right],
$$

where

$$
{ }_{2} F_{2}\left[a_{1}, a_{2} ; b_{1}, b_{2} ; x\right]=\sum_{k=0}^{\infty} \frac{\left(a_{1}\right)_{k}\left(a_{2}\right)_{k} x^{k}}{\left(b_{1}\right)_{k}\left(b_{2}\right)_{k} k !}
$$

is the generalized hypergeometric function with the coefficients defined as

$$
(\alpha)_{0}=1 ; \quad(\alpha)_{1}=\alpha ; \quad(\alpha)_{k}=\alpha(\alpha+1) \ldots(\alpha+k-1) \quad(k \geqslant 2) .
$$

The plot of $\tilde{E}$ versus $\theta$ as predicted by (3.11) is shown in figure 2. As follows from this figure, $\tilde{E}$ monotonically increases with increasing $\theta$, which indicates the dissipation of vortex ring energy with time. In the same figure, the plots obtained under the assumptions of small and large $\theta$ are shown. These will be discussed later in $\S 5$.

The form of (3.11) is exactly the same as in the case of conventional laminar vortex rings. However, the explicit time dependence of $\tilde{E}$ predicted by this equation is obviously different from that predicted by the conventional model due to the different functions $\ell(t)$. To illustrate this effect, let us assume that at a certain moment in time, $t_{0}, \theta\left(t_{0}\right) \equiv \theta_{0} \equiv R_{0} /\left(a t_{0}^{b}\right)=1$. Hence, at an arbitrary time, $t, \theta=\theta_{0}\left(t / t_{0}\right)^{-b}=\tilde{t}^{-b}$, where $\tilde{t}=t / t_{0}$. The plots of $\tilde{E}$ versus $\tilde{t}$ for $b=1 / 2$ and $1 / 4$ in the range $0 \leqslant \tilde{t} \leqslant 5$ are shown in figure 3 . As can be seen from figure 3 , the rate of energy decrease appears 


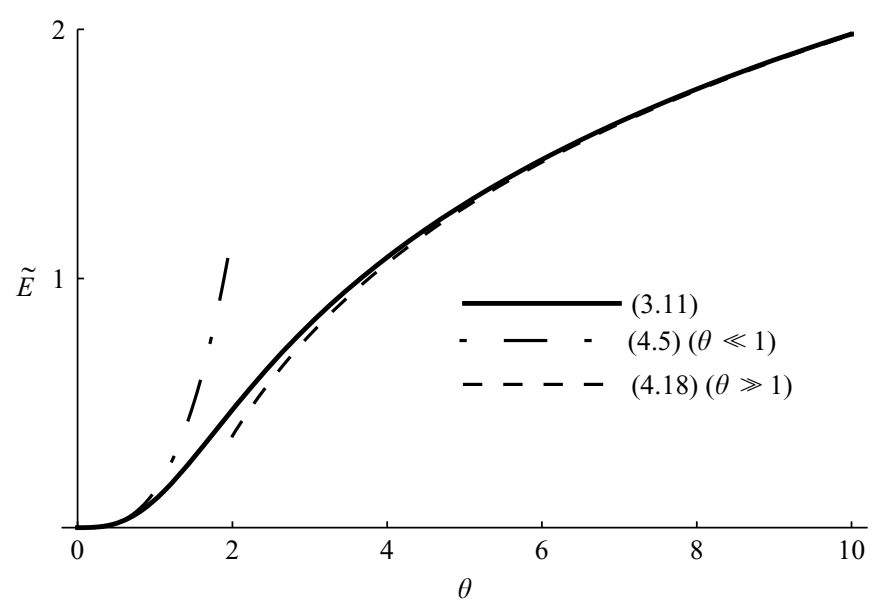

FIGURE 2. The plots of $\tilde{E}$ versus $\theta$ as predicted by (3.11) (arbitrary $\theta),(4.5)(\theta \ll 1)$ and (4.18) $(\theta \gg 1)$.

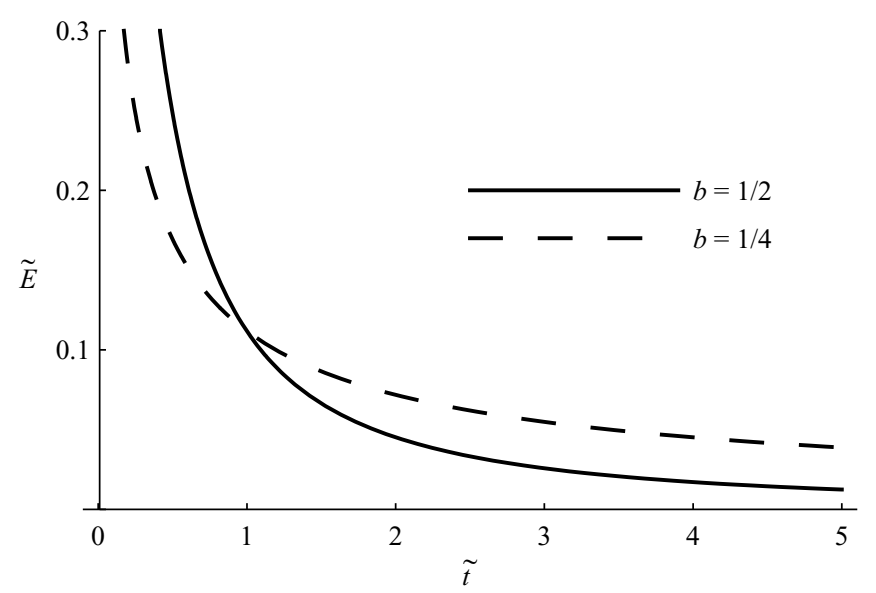

FIGURE 3 . The plots of $\tilde{E}$ versus $\tilde{t}=t / t_{0}$ as predicted by (3.11) for $b=1 / 2$ and $1 / 4$.

to be rather sensitive to the value of $b$. For $b=1 / 2$ (laminar case) this rate is the maximal one, while for $b=1 / 4$ this rate is the minimal one in the vicinity of $\tilde{t}=1$. At $\tilde{t}=1$ the plots for all $b$ coincide as expected.

Although the energy is an important parameter for vortex ring characteristics, it is difficult to measure it in practical applications. A more practically important characteristic of vortex rings is the translational velocity, introduced earlier (see (2.3)). Following Saffman (1970), this velocity is described in terms of the velocity of the vortex ring centroid $V_{x}$, calculated based upon the following general equation (Lamb 1932; Helmholtz 1858):

$$
V_{x}=\frac{\int_{0}^{\infty} \int_{-\infty}^{\infty}\left(\Psi-6 x r v_{r}\right) \zeta \mathrm{d} x \mathrm{~d} r}{\int_{0}^{\infty} \int_{-\infty}^{\infty} r^{2} \zeta \mathrm{d} x \mathrm{~d} r}
$$

As in the case of velocities $u_{x}$ and $u_{r}$, this velocity will be normalized by $v_{n}$. 


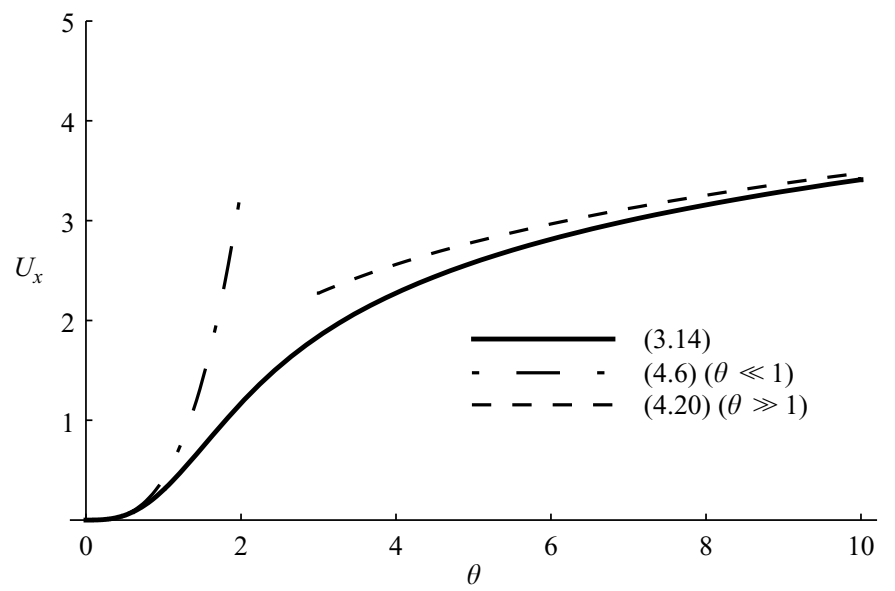

FIGURE 4. The plots of $U_{x}$ versus $\theta$ as predicted by (3.14) (arbitrary $\theta$ ), (4.6) $(\theta \ll 1)$ and (4.20) $(\theta \gg 1)$.

Using (2.4), (3.5) and (3.13), we obtain, following the approach developed by Kaplanski \& Rudi (2005)

$$
\begin{aligned}
U_{x}=\frac{V_{x}}{v_{n}}=\sqrt{\pi} \theta\left\{3 \exp \left(-\frac{\theta^{2}}{2}\right) I_{1}\left(\frac{\theta^{2}}{2}\right)\right. & +\frac{\theta^{2}}{12}{ }_{2} F_{2}\left[\frac{3}{2}, \frac{3}{2} ; \frac{5}{2}, 3 ;-\theta^{2}\right] \\
& \left.-\frac{3 \theta^{2}}{5}{ }_{2} F_{2}\left[\frac{3}{2}, \frac{5}{2} ; 2, \frac{7}{2} ;-\theta^{2}\right]\right\},
\end{aligned}
$$

where the generalized hypergeometric function ${ }_{2} F_{2}\left[a_{1}, a_{2} ; b_{1}, b_{2} ; x\right]$ was defined earlier (see (3.12)).

The plot of $U_{x}$ versus $\theta$ as predicted by (3.14) is shown in figure 4. As follows from this figure, $U_{x}$ monotonically increases with increasing $\theta$, as in the case of the vortex ring energy. In the same figure, the plots obtained for the assumptions of small and large $\theta$ are shown. These will be discussed later in $\S 4$.

As in the case of vortex ring energy, the form of (3.14) is exactly the same as for the case of conventional laminar vortex rings. However, the explicit time dependence of $U_{x}$ predicted by this equation differs from that predicted by the conventional model due to the different functions $\ell(t)$. As in the case of the vortex ring energy, we assume that at a certain moment in time, $t_{0}, \theta_{0} \equiv R_{0} /\left(a t_{0}^{b}\right)=1$. Hence, at an arbitrary $t$, $\theta=\theta_{0}\left(t / t_{0}\right)^{-b}=\tilde{t}^{-b}$, where $\tilde{t}=t / t_{0}$. The plots of $U_{x}$ versus $\tilde{t}$ for $b=1 / 4$ and $1 / 2$ in the same range of $\theta$ as in figure 3 are shown in figure 5 .

As can be seen from figure 5 , the velocity $U_{x}$ is a monotonically decreasing function of time for all $b$, as in the case of the vortex ring energy. Also, similar to the vortex ring energy, the velocity decreases with time at a greater rate for larger $b$ when $\tilde{t}$ is close to 1 . As in the case of vortex ring energy, at $\tilde{t}=1$, the plots for all $b$ coincide as expected. The values of $U_{x}$ increase with increasing $b$ at $\tilde{t}<1$ and decrease with increasing $b$ at $\tilde{t}>1$. At $\tilde{t}>5, U_{x}$ decreases slowly with increasing $\tilde{t}$, remaining positive, as in the case of $\tilde{E}$.

Note that velocity $U_{x}$ coincides with the absolute velocity of the points at which $u_{x}=u_{r}=0$ (zero velocity in the moving frame of reference). Also, it was useful to introduce an additional velocity which described the movement of the points of maximal vorticity of vortex rings $\left(U_{\omega x}\right)$. As follows from (3.5), the $x$ coordinates of these points correspond to $\eta=\eta_{\max }=0$ or $x=x_{0}$. The $r$ coordinates of these points, 


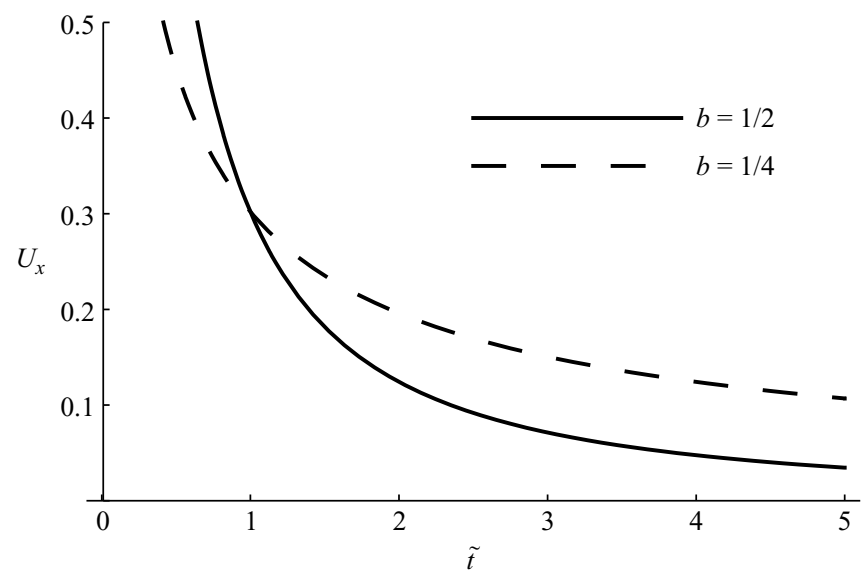

FIGURE 5. The plots of $U_{x}$ versus $\tilde{t}=t / t_{0}$ as predicted by (3.14) for $b=1 / 2$ and $1 / 4$.

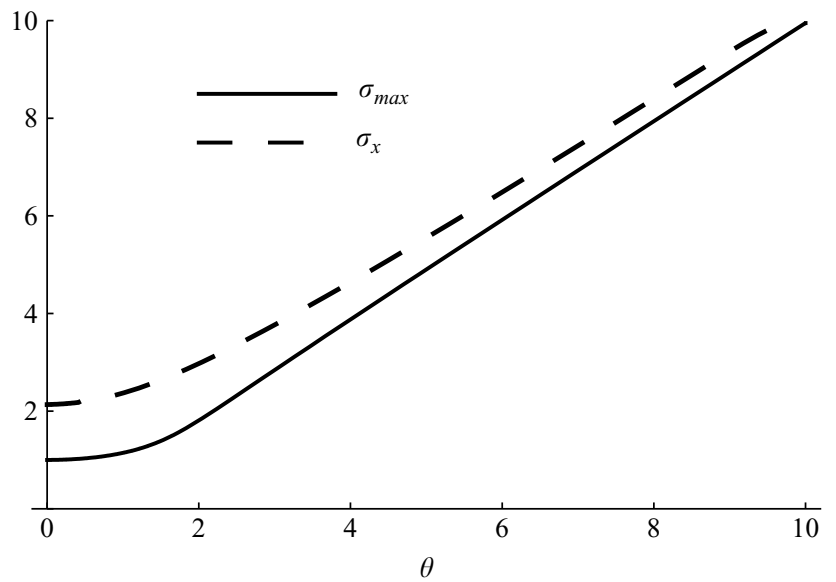

FIGURE 6. The plots of $\sigma_{\max }$ (location of the point of maximal vorticity) and $\sigma_{x}$ (location of the point at which $u_{x}=u_{r}=0$ ) versus $\theta$.

$\sigma=\sigma_{\max }$, can be found from the condition $\mathrm{d} \omega / \mathrm{d} \sigma=0$ which is translated into the following condition:

$$
\left(\sigma_{\max }^{2}+1\right) I_{1}\left(\sigma_{\max } \theta\right)=\sigma_{\max } \theta I_{0}\left(\sigma_{\max } \theta\right) .
$$

The plot of $\sigma_{\max }$ versus $\theta$ in the range of $\theta$ between 0 and 10 is shown in figure 6 . In the same figure, the plot of $\sigma_{x}$ (when $u_{x}$ changes sign) versus $\theta$ is shown. As follows from this figure, both $\sigma_{\max }$ and $\sigma_{x}$ monotonically increase with increasing $\theta$. The fact that $\sigma_{\max }$ is always less than $\sigma_{x}$ indicates that the region of maximal vorticity is always closer to the axis of the vortex ring than the region in which $u_{x}$ changes sign.

For large $\theta$, both plots approach the line $\sigma=\theta$ which corresponds to $r=R_{0}$. For $\theta \rightarrow 0, \sigma_{\max }$ approaches 1 from above, whilst $\sigma_{x}$ approaches 2 from above. The limiting values of $\sigma_{\max }$ will be discussed in $\S 4$. There is no simple explanation of the properties of $\sigma_{x}$, which were inferred from the numerical analysis of (3.8). 


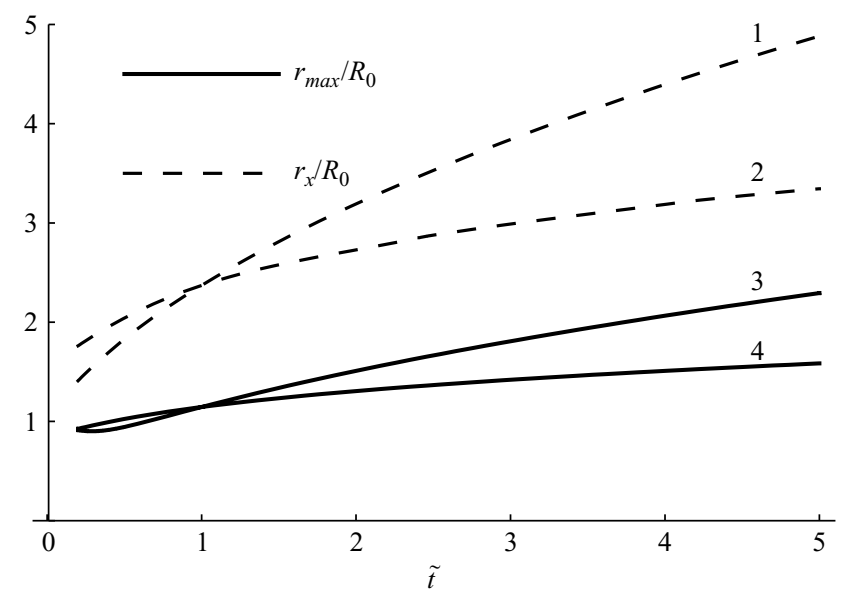

FIGURE 7. The plots of $r_{x} / R_{0}$ (location of the point at which $u_{x}=u_{r}=0$ ) versus $\tilde{t}=t / t_{0}$ for $b=1 / 2$ (curve 1 ) and $b=1 / 4$ (curve 2); the plots $r_{\max } / R_{0}$ (location of the point of maximal vorticity) versus $\tilde{t}=t / t_{0}$ for $b=1 / 2$ (curve 3 ) and $b=1 / 4$ (curve 4 ).

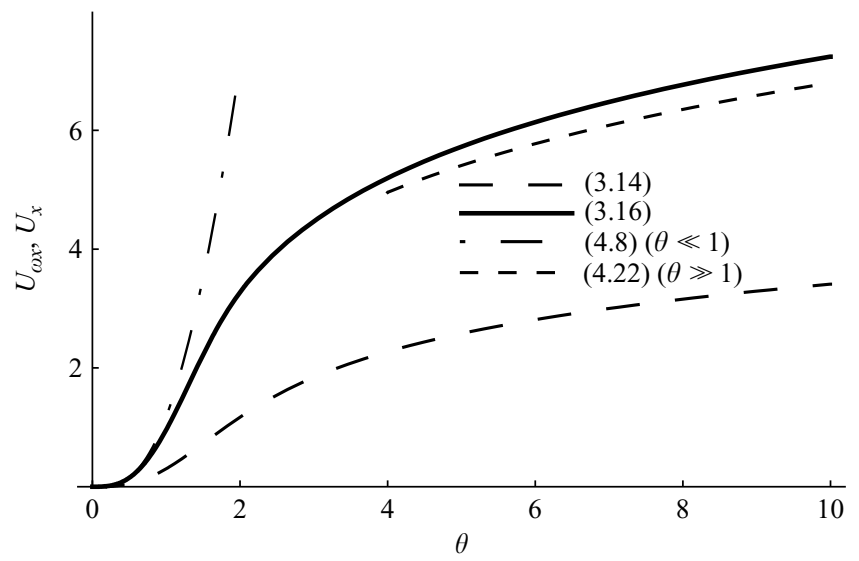

FIGURE 8. The plots of $U_{x}$ versus $\theta$ as predicted by (3.14) (long dashed curve), $U_{\omega x}$ versus $\theta$ as predicted by (3.16) for arbitrary $\theta$ (solid curve), (4.8) for small $\theta$ (dashed-dotted curve) and (4.22) for large $\theta$ (short dashed curve).

As in the case of figures 3 and 5 , we assume that $\theta_{0} \equiv R_{0} /\left(a t_{0}^{b}\right)=1$ which implies that $\theta=\tilde{t}^{-b}$. The plots of $r_{\max } / R_{0}=\sigma_{\max } / \theta$ and $r_{x} / R_{0}=\sigma_{x} / \theta$ versus $\tilde{t}$ for $b=1 / 2$ and $1 / 4$ in the range $0 \leqslant \tilde{t} \leqslant 5$ are shown in figure 7 . As follows from this figure, $r_{\max }$ is close to $R_{0}$ for all $b$ and $\tilde{t} \leqslant 1$. For $\tilde{t}>1, r_{\max }$ increases with increasing $\tilde{t}$ and increasing $b$. In agreement with figure $6, r_{x}$ is always greater than $r_{\max }$.

Remembering (3.8) we obtain the expression for the normalized axial velocity of fluid in the region of maximal vorticity in the form

$$
U_{\omega x} \equiv V_{\omega x} / v_{n}=U_{x}+2 \pi \theta^{2} \int_{0}^{\infty} \mu \operatorname{erfc}\left(\frac{\mu}{\sqrt{2}}\right) J_{1}(\theta \mu) J_{0}\left(\sigma_{\max } \mu\right) \mathrm{d} \mu,
$$

where $\theta=\theta_{\max }$ satisfies (3.15).

The plots of $U_{\omega x}$ and $U_{x}$ versus $\theta$ in the range of $\theta$ between 0 and 10 are shown in figure 8. As can be seen from this figure, both $U_{\omega x}$ and $U_{x}$ increase with increasing $\theta$; 


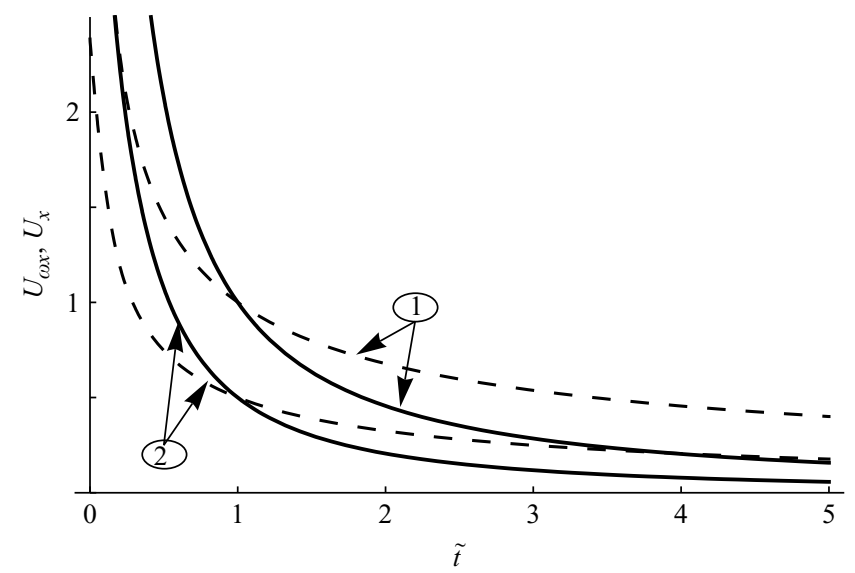

FIGURE 9. The plots of $U_{\omega x}$ (predicted by (3.16); curves 1) and $U_{x}$ (predicted by (3.14); curves 2 ) versus $\tilde{t}$ for $b=1 / 2$ (solid curves) and $1 / 4$ (dashed curves).

$U_{\omega x}$ is always substantially greater than $U_{x}$, especially at $\theta>1$. In the same figure, the plots of $U_{\omega x}$ and $U_{x}$ versus $\theta$ obtained under the assumptions that $\theta \ll 1$ and $\theta \gg 1$ are shown. These are discussed later in $\S 4$.

As in the case of figures 3,4 and 7 , it is assumed that $\theta_{0} \equiv R_{0} /\left(a t_{0}^{b}\right)=1$ which implies that $\theta=\tilde{t}^{-b}$. The plots of $U_{\omega x}$ and $U_{x}$ versus $\tilde{t}$ for $b=1 / 2$ and $1 / 4$ in the range $0 \leqslant \tilde{t} \leqslant 5$ are shown in figure 9. Both $U_{\omega x}$ and $U_{x}$ decrease with increasing time, the values of $U_{\omega x}$ being always greater than the values of $U_{x}$, in agreement with figure 8. At $\tilde{t}=1$, both $U_{\omega x}$ and $U_{x}$ do not depend on $b$ as in the cases shown in figures 3,5 and 7 .

From (3.9) it can be seen that the predicted radial component of velocity at the points of maximal vorticity of vortex rings $(\eta=0)$ is equal to zero. This is an expected result, as the streamlines at $\eta=0$ are always perpendicular to plane $\eta=0$. However, this zero fluid velocity in the $r$ direction by no means prohibits the movement of the point corresponding to the maximal vorticity $\left(\eta_{\max }, \sigma_{\max }\right)$ in this direction. The dimensionless effective radial velocity of this point can be found from (3.15) such that

$$
U_{e f f(r)}=\frac{1}{v_{n}} \frac{\mathrm{d} r_{\max }}{\mathrm{d} t}
$$

where $r_{\max }=\ell \sigma_{\max }$.

Note that in contrast to the previously calculated velocities, the expression for $U_{\text {eff } r}$ contains an additional parameter $M$, via $v_{n}$. This makes it difficult to compare directly the values of $U_{\omega x}$ predicted by (3.16) and the values of $U_{\text {eff }(r)}$ predicted by (3.17).

As in the case of figures $3,4,7$ and 9 , it is assumed that $\theta\left(t_{0}\right)=\theta_{0}=1$. In this case, $\theta=\left(t / t_{0}\right)^{-b}=\tilde{t}^{-b}$. Also, we assume that $v_{n}=1 \mathrm{~m} \mathrm{~s}^{-1}$ and $a=1 \mathrm{~m} \mathrm{~s}^{-b}$. The plots of $U_{\text {eff(r) }}$ versus $\tilde{t}$ are shown in figure 10. As can be seen from this figure, at short times $(\tilde{t}<1)$ the time dependence of $U_{\text {eff }(r)}$ is complex and highly depends upon the value of $b$. This will be discussed in more detail in $\S 4$. However, at long times (starting from approximately $\tilde{t}=1) U_{\text {eff }(r)}$ is a very slowly decreasing function of time. The values of $U_{\text {eff }(r)}$ at these times decrease with decreasing $b$.

One of the important limitations of the model described so far is that it is based upon the assumption that $R_{0}=$ constant. The approach suggested by Saffman (1970) and further developed by Weigand \& Gharib (1997) for laminar vortex rings can be used to generalize our model to the case of non-constant $R_{0}$, which will be referred 


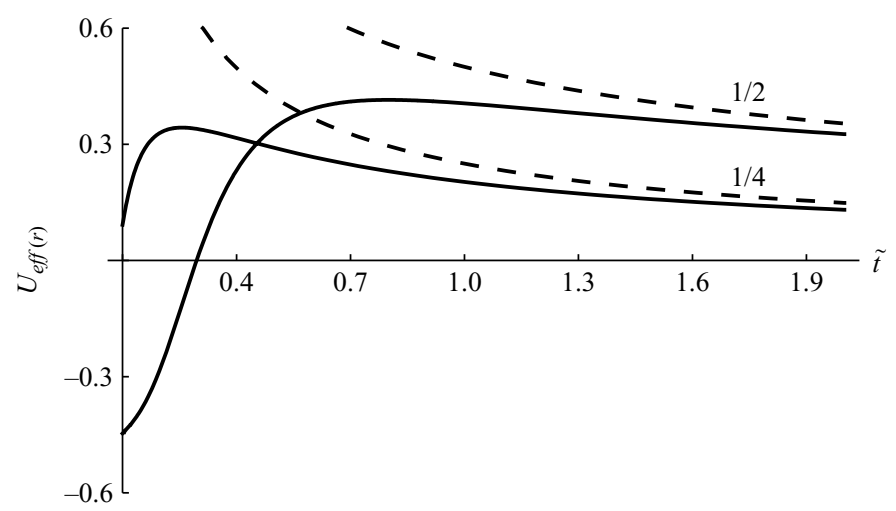

Figure 10. The plots of $U_{\text {eff }(r)}$ versus $\tilde{t}$ predicted by (3.17) (arbitrary $\tilde{t}$; solid curves) and (4.9) (large $\tilde{t}$; dashed curves) for $b=1 / 2$ and $1 / 4$ (numbers near the curves).

to as $R$. We start with the dimensionally correct equation

$$
V_{x}=\frac{M}{k R^{3}},
$$

where $k$ is a proportionality constant. The decay of circulation can be described by the second dimensionally correct equation

$$
\frac{\mathrm{d}\left(V_{x} R\right)}{\mathrm{d} t}=-k^{\prime} \frac{\nu_{*} V_{x}}{R}
$$

where $k^{\prime}$ is another proportionality constant. The viscosity $v_{*}$ is defined by (2.7). In contrast to the case considered by Saffman (1970) and Weigand \& Gharib (1997), $v_{*}$ depends on time. Having substituted (2.7) into (3.19) and integrating the latter equation from $t=t_{0}=0$ to $t$, one obtains

$$
R^{2}-R_{0}^{2}=\frac{k^{\prime} a^{2}}{2} t^{2 b}
$$

For $b=1 / 2$ and $a=\sqrt{2 v},(3.20)$ reduces to the one derived by Saffman (1970) and Weigand \& Gharib (1997). Substituting (3.20) into (3.18) gives

$$
V_{x}=\frac{M}{k\left(R_{0}^{2}+\frac{k^{\prime} a^{2}}{2} t^{2 b}\right)^{3 / 2}} .
$$

For $b=1 / 2$ and $a=\sqrt{2 v},(3.20)$ and (3.21) reduce to the corresponding equations derived by Saffman (1970) and Weigand \& Gharib (1997). In the dimensionless form, (3.21) can be rewritten as

$$
U_{x}=\frac{4 \pi^{2}}{k\left(1+\frac{k^{\prime}}{2 \theta^{2}}\right)^{3 / 2}} .
$$

The form of (3.22) depends neither upon $a$ nor upon $b$. As in the previous cases, it is assumed that $\theta\left(t_{0}\right)=\theta_{0}=1$. In this case, $\theta=\tilde{t}^{-b}$, and (3.22) can be rewritten as follows:

$$
U_{x}=\frac{4 \pi^{2}}{k\left(1+\frac{k^{\prime} \tau^{2 b}}{2}\right)^{3 / 2}} .
$$

Equations (3.20)-(3.23) can be considered the generalization of the so-called Saffman's second formula (see (1.3)) for the vortex ring velocity for arbitrary $a$ and $b$. 


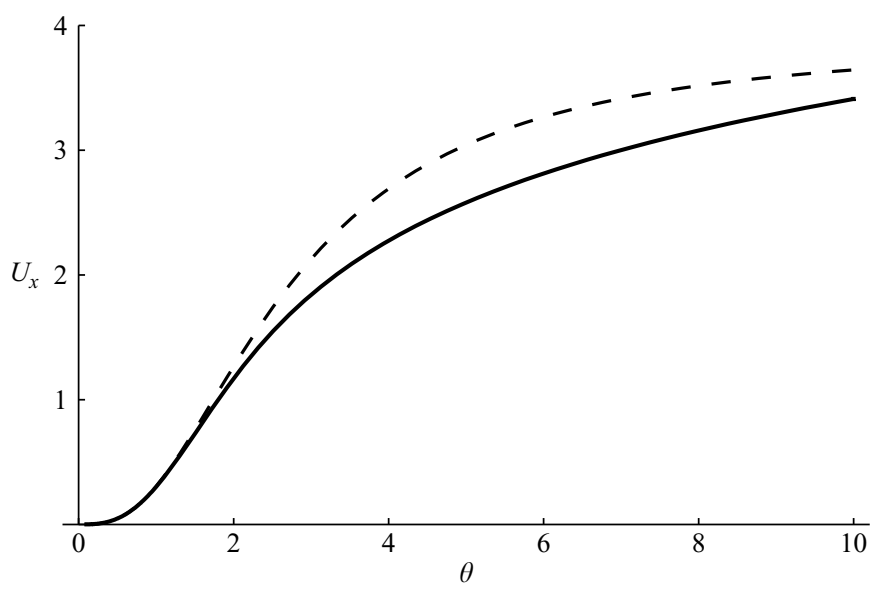

FIGURE 11. The plots of $U_{x}$ versus $\theta$ based on (3.14) (solid curve) and (3.23) (dashed curve) for $k=10.153200$ and $k^{\prime}=8.9090909$.

The values of $k$ and $k^{\prime}$ could be obtained based upon the best fit with the experimental data of Weigand \& Gharib (1997). Alternatively they can be obtained based on the minimal deviation of $U_{x}$ predicted by (3.23) and (3.14) in the limit of long $t$. As the criterion of this minimal deviation, one can use the coincidence of the first two terms of the asymptotic expansions of these equations in the limit of long times. This leads to the following values:

$$
k=\frac{1320}{2401} \sqrt{11} \pi^{3 / 2} \approx 10.153200, \quad k^{\prime}=\frac{98}{11} \approx 8.9090909 .
$$

The plots of $U_{x}$ versus $\theta$ based on (3.14) and (3.23) for these values of $k$ and $k^{\prime}$ are shown in figure 11. A reasonable agreement between the values of $U_{x}$ predicted by these equations is observed over the whole range of $\theta$. At $\theta<2$ these values approximately coincide as expected.

Let us now introduce another dimensionless time defined as

$$
t^{*}=\frac{a^{2} t^{2 b}}{32 R_{0}^{2}}=\frac{1}{32 \theta^{2}} \text {. }
$$

In the laminar case, when $a=\sqrt{2 v}$ and $b=1 / 2, t^{*}$ reduces to the one introduced by Weigand \& Gharib (1997). Remembering (3.24), (3.22) can be rewritten as

$$
U_{x}=\frac{4 \pi^{2}}{k\left(1+16 k^{\prime} t^{*}\right)^{3 / 2}} .
$$

This equation will be investigated in $\S 5$.

\section{Limiting cases}

In this section the limiting cases of the solutions presented in $\S 3$, referring to long and short times, will be discussed. 


\subsection{Long times}

In the long time limit $\theta \ll 1,(3.5)$ and (3.6) can be simplified to

$$
\begin{aligned}
& \omega=\frac{\sigma \theta}{2} \exp \left[-\frac{1}{2}\left(\sigma^{2}+\eta^{2}\right)\right], \\
& \Phi=\frac{\sigma \sqrt{2 \pi} \theta}{16} \int_{0}^{\infty} \mu F(\mu, \eta) J_{1}(\sigma \mu) \mathrm{d} \mu .
\end{aligned}
$$

Unfortunately, the latter integral cannot be presented in an analytical form. An alternative calculation of $\Phi$ can be based on substitution of the expression (4.1) into (2.4). The solution of the latter equation gives (Phillips 1956)

$$
\Phi=\frac{\theta \sqrt{\pi}}{2 \sqrt{2}} \frac{\sigma^{2}}{\left(\sigma^{2}+\eta^{2}\right)^{3 / 2}}\left[\operatorname{erf}\left(s_{*}\right)-\frac{2 s_{*}}{\sqrt{\pi}} \exp \left(-s_{*}^{2}\right)\right],
$$

where

$$
s_{*}=\sqrt{\frac{\sigma^{2}+\eta^{2}}{2}} .
$$

Note that although $\omega$ predicted by (4.1) depends upon $\theta$, the corresponding formula for the dimensional vorticity does not contain $R_{0}$. This leads to a self-similar solution when the vorticity depends upon only one parameter, the vortex ring momentum $M$ (cf. Lugovtsov 1970, 1976).

The combination of this equation and (2.3) leads to the following expressions for the velocity components:

$$
\begin{gathered}
u_{x}=\frac{\sqrt{2 \pi} \theta^{3}}{2\left(\sigma^{2}+\eta^{2}\right)^{5 / 2}} \exp \left(-\frac{\sigma^{2}+\eta^{2}}{2}\right)\left[2 \sqrt{\sigma^{2}+\eta^{2}}\left(\sigma^{4}-2 \eta^{2}+\sigma^{2}\left(1+\eta^{2}\right)\right)\right. \\
\left.-\sqrt{2 \pi} \exp \left(\frac{\sigma^{2}+\eta^{2}}{2}\right)\left(\sigma^{2}-2 \eta^{2}\right) \operatorname{erf}\left(\frac{\sqrt{\sigma^{2}+\eta^{2}}}{\sqrt{2}}\right)\right], \\
u_{r}=-\frac{\sqrt{2 \pi} \sigma \eta \theta^{3}}{2}\left\{\frac{2 \exp \left(-\frac{\sigma^{2}+\eta^{2}}{2}\right)\left(3+\sigma^{2}+\eta^{2}\right)}{\left(\sigma^{2}+\eta^{2}\right)^{2}}-\frac{3 \sqrt{2 \pi} \operatorname{erf}\left(\frac{\sqrt{\sigma^{2}+\eta^{2}}}{\sqrt{2}}\right)}{\left(\sigma^{2}+\eta^{2}\right)^{5 / 2}}\right\} .
\end{gathered}
$$

Keeping only the zeroth term in series (3.12), (3.11) and (3.14) are simplified to

$$
\begin{aligned}
\tilde{E} & =\frac{\sqrt{\pi} \theta^{3}}{12}, \\
U_{x} & =\frac{7 \sqrt{\pi} \theta^{3}}{30} .
\end{aligned}
$$

This dimensionless velocity corresponds to

$$
V_{x}=\frac{7}{120 \pi \sqrt{\pi}} \frac{M}{a^{3}} t^{-3 b} \approx 0.0105 \frac{M}{a^{3}} t^{-3 b} .
$$

The plot of $\tilde{E}$ versus $\theta$, based on (4.5), is shown in figure 2. As follows from this figure, at $\theta<1 / 2$ the values of $\tilde{E}$ predicted by (4.5) show very close agreement with those predicted by (3.11). 


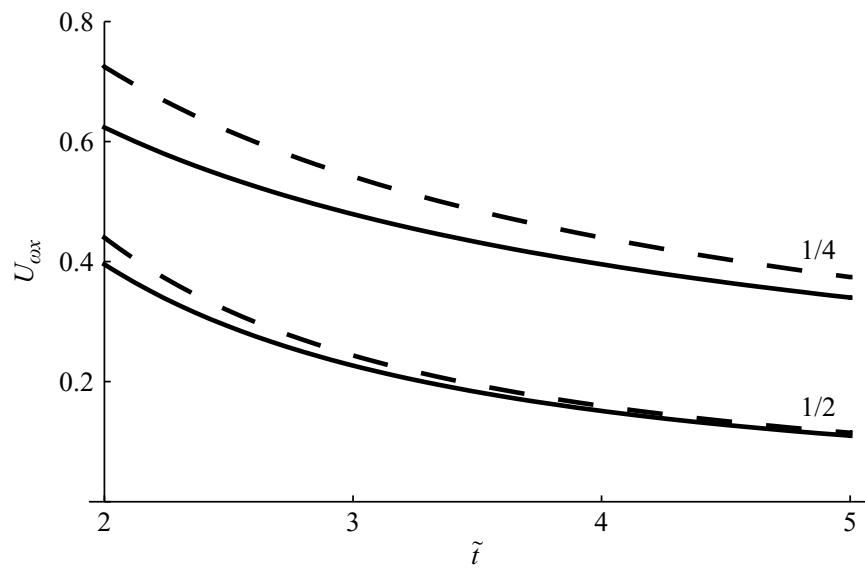

FiguRE 12. The plots of $U_{\omega x}$ versus $\tilde{t}$ as predicted by the general equation (3.16) (solid curves) and the simplified equation (4.8) (dashed curves) for $b=1 / 2$ and $1 / 4$ (numbers near the curves).

The plot of $U_{x}$ versus $\theta$, based on (4.6), is shown in figure 4. As follows from this figure, at $\theta<1 / 2$, the values of $U_{x}$ predicted by (4.6) are again almost indistinguishable from those predicted by (3.14), as in the case of the vortex ring energy.

For $a=\sqrt{2 v}$ and $b=1 / 2,(4.7)$ is identical to the one obtained by Rott $\&$ Cantwell (1993a) (see (1.2)). For $b=1 / 4$ the time dependence of $V_{x}$ is identical to the one reported earlier by Lugovtsov (1976), Glezer \& Coles (1990), Cantwell (2002) and Afanasyev \& Korabel (2004).

The location of the point of the maximal vorticity in the limit of small $\theta\left(\eta_{\max }=\right.$ $0, \sigma_{\max }=1$ ) follows from (3.15) (the latter condition corresponds to $r=\ell$ ). In this case, (3.16) is simplified to

$$
U_{\omega x}=\frac{7 \sqrt{\pi} \theta^{3}}{30}+2 \pi \theta^{2} \int_{0}^{\infty} \mu \operatorname{erfc}\left(\frac{\mu}{\sqrt{2}}\right) J_{1}(\theta \mu) J_{0}(\mu) \mathrm{d} \mu .
$$

When deriving (4.8) it is important to note that in the limit $\theta \ll 1, U_{x}$ is given by (4.6).

The plots of $U_{\omega x}$ versus $\theta$ based on (4.8) are shown in figure 8 . For $\theta<1$, the values of $U_{\omega x}$ predicted by (4.8) are very close to those predicted by (3.16), as in the case of $U_{x}$ (see figure 4).

As in the case of figures 3, 4, 7 and 9, it is assumed once again that $\theta_{0} \equiv R_{0} /\left(a t_{0}^{b}\right)=1$ which implies that $\theta=\tilde{t}^{-b}$. The plots of $U_{\omega x}$ versus $\tilde{t}$ for $b=1 / 2$ and $1 / 4$, predicted by (3.16) and (4.8) in the range $2 \leqslant \tilde{t} \leqslant 5$ are shown in figure 12 . The values of $U_{\omega x}$ predicted by (3.16) and (4.8) are reasonably close for all $b$ in the whole range of $\tilde{t}$ under consideration, although the closeness of the curves deteriorates with decreasing $b$.

As already mentioned, in a long time limit $(\theta \ll 1)$, the solution of $(3.15)$ can be presented as $\sigma_{\max }=1$ which corresponds to $r_{\max }=\ell$. In this case, the dimensionless effective radial velocity of this point can be found from (3.15) in the form

$$
U_{\text {eff }(r)}=\frac{1}{v_{n}} \frac{\mathrm{d} r_{\text {max }}}{\mathrm{d} t}=\frac{1}{v_{n}} \frac{\mathrm{d} \ell}{\mathrm{d} t}=\frac{a b t^{b-1}}{v_{n}} .
$$

Assuming that $\theta_{0} \equiv R_{0} /\left(a t_{0}^{b}\right)=1, \theta=\tilde{t}^{-b}$. The plots of $U_{e f f(r)}$ versus $\tilde{t}$ for $b=1 / 2$ and 1/4, predicted by (4.9) (for $v_{n}=1 \mathrm{~m} \mathrm{~s}^{-1}$ and $a=1 \mathrm{~m} \mathrm{~s}^{-b}$ ) are shown in figure 10, 


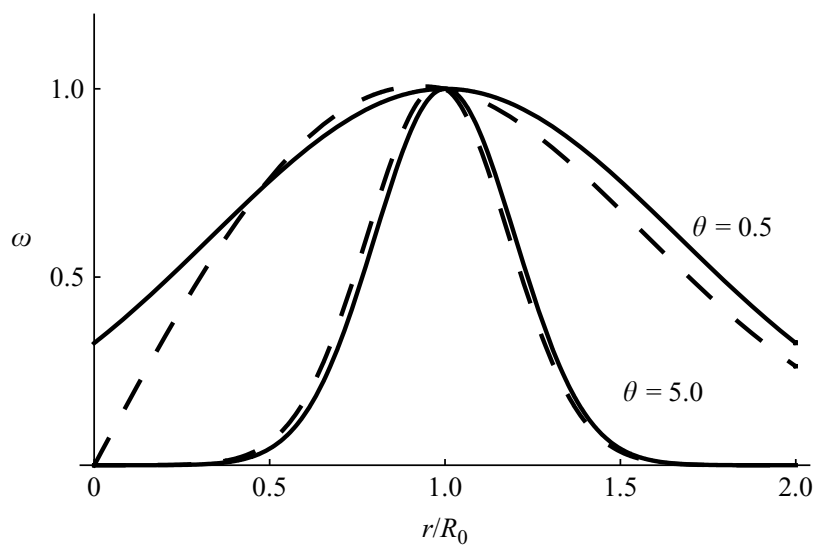

FIGURE 13. The plots of normalized $\omega$ versus $r / R_{0}$ as predicted by (3.5) (dashed curves) and (4.11) (solid curves) for $\theta=5$ and $\theta=0.5$ (numbers near the curves).

alongside the corresponding curves predicted by (3.17). As follows from this figure, the values of $U_{\text {eff }(r)}$ predicted by (3.17) and (4.9) are reasonably close for all $b$ and $\tilde{t}>1$.

Note that in the case of $\theta \rightarrow 0$ we have $\ell \gg R_{0}$. In this case the vortex ring looses its conventional torus form, and it might be ambiguous to call it a cohesive ring.

\subsection{Short times}

In a short time limit $(\theta \gg 1)$, (3.5) can be simplified to

$$
\omega=\sqrt{\frac{1}{2 \pi \sigma \theta}} \exp \left[-\frac{1}{2}\left((\sigma-\theta)^{2}+\eta^{2}\right)\right]\left(1-\frac{3}{8 \sigma \theta}-\ldots\right) .
$$

Having introduced a new dimensionless parameter $s$ as the dimensionless distance from the point $\left(R_{0} / \ell, x_{0} / \ell\right)$ and assuming that $\left|R_{0}-r\right| \ll R_{0}$, (4.10) can be further simplified to (see Kaplanski \& Rudi 1999)

$$
\omega=\frac{1}{\sqrt{2 \pi} \theta} \exp \left(-\frac{1}{2} s^{2}\right)\left(1+O\left(\frac{\left|R_{0}-r\right|}{2 R_{0}}\right)\right) .
$$

This equation is identical to the one reported earlier by Wang, Chu \& Chien-Chang (1997). It predicts the Gaussian distribution with respect to $r / R_{0}$.

The plots of $\omega$ versus $r / R_{0}$ predicted by (3.5) and (4.11) are compared in figure 13 for $\theta=5$ and $\theta=0.5$. These plots almost coincide for short times $(\theta=5)$ but show the greatest deviation for long times $(\theta=0.5)$.

Assuming that $\eta \gg 1$, one obtains

$$
\begin{aligned}
& \operatorname{erfc}\left(\frac{\mu-\eta}{\sqrt{2}}\right) \rightarrow 2, \\
& \operatorname{erfc}\left(\frac{\mu+\eta}{\sqrt{2}}\right) \rightarrow \frac{\sqrt{2} \exp \left(-\frac{(\mu+\eta)^{2}}{2}\right)}{(\mu+\eta) \sqrt{2}} .
\end{aligned}
$$

The latter relation means that erfc $((\mu+\eta) / \sqrt{2})$ approaches zero faster than $\exp (-\mu \eta)$ for large $\eta$. A similar analysis can be performed for $\eta \ll-1$. Hence, (3.7) can be 
simplified to

$$
\Phi=\frac{\sigma \sqrt{2 \pi}}{2} \int_{0}^{\infty} \exp (-\mu|\eta|) J_{1}(\theta \mu) J_{1}(\sigma \mu) \mathrm{d} \mu .
$$

This equation is similar to the one given by (Lamb 1932, (14), p. 239).

Using the same assumptions as during the derivation of (4.12), the following equations for the normalized components of velocity are obtained:

$$
\begin{aligned}
& u_{x}=\pi \theta^{2} \int_{0}^{\infty} \mu \exp (-\mu|\eta|) J_{1}(\theta \mu) J_{0}(\sigma \mu) \mathrm{d} \mu, \\
& u_{r}=-\pi \theta^{2} \int_{0}^{\infty} \mu \exp (-\mu|\eta|) J_{1}(\theta \mu) J_{1}(\sigma \mu) \mathrm{d} \mu .
\end{aligned}
$$

In the limit of large $x$, the following asymptotic representation of the generalized hypergeometric function will be used (Mathematica 2007):

$$
\begin{aligned}
{ }_{2} F_{2}\left[a_{1}, a_{2} ; b_{1}, b_{2} ; x\right]= & {\left[\frac{\exp (x) x^{a_{1}+a_{2}-b_{1}-b_{2}} \Gamma\left(b_{1}\right) \Gamma\left(b_{2}\right)}{\Gamma\left(a_{1}\right) \Gamma\left(a_{2}\right)}+\frac{(-x)^{-a_{1}} \Gamma\left(a_{2}-a_{1}\right) \Gamma\left(b_{1}\right) \Gamma\left(b_{2}\right)}{\Gamma\left(a_{2}\right) \Gamma\left(b_{1}-a_{1}\right) \Gamma\left(b_{2}-a_{1}\right)}\right.} \\
& \left.+\frac{(-x)^{-a_{2}} \Gamma\left(a_{1}-a_{2}\right) \Gamma\left(b_{1}\right) \Gamma\left(b_{2}\right)}{\Gamma\left(a_{1}\right) \Gamma\left(b_{1}-a_{2}\right) \Gamma\left(b_{2}-a_{2}\right)}\right]+O\left(\frac{1}{x}\right)
\end{aligned}
$$

when $a_{1} \neq a_{2}$, and

$$
\begin{aligned}
& { }_{2} F_{2}\left[a_{1}, a_{1} ; b_{1}, b_{2} ; x\right]=\left[\frac{\exp (x) x^{2 a_{1}-b_{1}-b_{2}} \Gamma\left(b_{1}\right) \Gamma\left(b_{2}\right)}{\Gamma^{2}\left(a_{1}\right)}\right. \\
& \left.+\frac{(-x)^{-a_{1}} \Gamma\left(b_{1}\right) \Gamma\left(b_{2}\right)\left[-2 \gamma+\log (-x)-\psi\left(a_{1}\right)-\psi\left(b_{1}-a_{1}\right)-\psi\left(b_{2}-a_{1}\right)\right]}{\Gamma\left(a_{1}\right) \Gamma\left(b_{1}-a_{1}\right) \Gamma\left(b_{2}-a_{1}\right)}\right] \\
& \quad+O\left(\frac{1}{x}\right),
\end{aligned}
$$

where $\gamma \approx 0.57721566$ is the Euler constant; $\Gamma(x)$ is the Gamma function; and $\psi(x)$ is the di-gamma function defined as

$$
\psi(x)=\frac{\mathrm{d} \log \Gamma(x)}{\mathrm{d} x} .
$$

Having substituted (4.16) into (3.11) we obtain

$$
\tilde{E}=\ln (\theta)-\gamma / 2-\psi(3 / 2) .
$$

When deriving (4.18) the contribution of the imaginary term in (4.16) is ignored, and the expression for ${ }_{2} F_{2}$ is rewritten for the required values of parameters as

$$
{ }_{2} F_{2}\left[\frac{3}{2}, \frac{3}{2} ; \frac{5}{2}, 3 ;-\theta^{2}\right]=\frac{12 \theta^{-3}}{\sqrt{\pi}}\left[\ln \theta-\frac{\gamma}{2}-\psi\left(\frac{3}{2}\right)\right] .
$$

Note than $\psi(1)=\gamma$.

Remembering that

$$
\frac{\gamma}{2}+\psi(3 / 2) \approx \frac{1}{2}+2.058-\ln 8
$$

Equation (4.18) is identical to the one derived by Saffman (1992).

The plot of $\tilde{E}$ versus $\theta$, based on (4.18), is shown in figure 2. As follows from this figure, at $\theta>5$ the values of $\tilde{E}$ predicted by (4.18) almost coincide with those predicted by (3.11). 
Having substituted (4.15) and (4.16) into (3.14) one obtains

$$
U_{x}=\ln \theta+\frac{3-\gamma}{2}-\psi(3 / 2)+O\left(\frac{1}{\theta}\right) .
$$

When deriving (4.20), (4.15) was rewritten for the required values of parameters as

$$
{ }_{2} F_{2}\left[\frac{3}{2}, \frac{5}{2} ; \frac{5}{2}, 3 ;-\theta^{2}\right]=\frac{5 \theta^{-3}}{2 \sqrt{\pi}},
$$

and it was taken into account that

$$
3 \exp \left(-\frac{\theta^{2}}{2}\right) I_{1}\left(\frac{\theta^{2}}{2}\right)=\frac{3}{\sqrt{\pi} \theta} .
$$

In the case in which $\ell=\sqrt{2 v t}$, (4.20) reduces to the one obtained by Saffman (1970) (see (1.1)).

The plot of $U_{x}$ versus $\theta$, based on (4.20), is shown in figure 4. As follows from this figure, at $\theta>5$ the values of $U_{x}$ predicted by (4.20) show good agreement with those predicted by (3.14); the difference between the values of $U_{x}$ predicted by these equations is clearly visible over the whole range of $\theta$ under consideration.

The location of the point of the maximal vorticity $\left(\eta_{\max }=0, \sigma_{\max }=\theta\right)$ follows from (3.15) (with the latter condition corresponding to $r=R_{0}$ ). In this case, (3.16) is simplified to

$$
U_{\omega x}=\ln \theta+\frac{3-\gamma}{2}-\psi(3 / 2)+2 \pi \theta^{2} \int_{0}^{\infty} \mu \operatorname{erfc}\left(\frac{\mu}{\sqrt{2}}\right) J_{1}(\theta \mu) J_{0}(\theta \mu) \mathrm{d} \mu .
$$

When deriving (4.22) it was considered that in the limit $\theta \gg 1, U_{x}$ is given by (4.20).

The plots of $U_{\omega x}$ versus $\theta$ based on (4.22) are shown in figure 8. As follows from this figure, for $\theta>1$ the values of $U_{\omega x}$ predicted by (4.22) are reasonably close to the ones predicted by (3.16), although the closeness of the corresponding curves is worse than in the case of $U_{x}$ (see figure 4).

As in the case of figures $3,4,7,9$ and 12 , it is assumed that $\theta_{0} \equiv R_{0} /\left(a t_{0}^{b}\right)=1$ which implies that $\theta=\tilde{t}^{-b}$. The plots of $U_{\omega x}$ versus $\tilde{t}$ for $b=1 / 2$ and $1 / 4$, predicted by (3.16) and (4.22) for $\tilde{t} \leqslant 1$ are shown in figure 14. The values of $U_{\omega x}$ predicted by (3.16) and (4.22) are reasonably close for all $b$ for sufficiently small $\tilde{t}$. Note that the range of closeness of the curves shown in figure 14 is outside the range shown in figure 8 .

In a short time limit $(\theta \gg 1),(3.15)$ can be simplified to

$$
\left(\sigma_{\max }^{2}+1\right)\left(1-\frac{3}{8 \sigma_{\max } \theta}\right)=\sigma_{\max } \theta\left(1-\frac{1}{8 \sigma_{\max } \theta}\right) .
$$

This is an algebraic equation with respect to $\theta$. Its physically meaningful solution can be presented as

$$
\theta=\sigma_{\text {max }}+\frac{3}{4 \sigma_{\max }} .
$$

In the dimensional form this solution can be presented as

$$
r_{\text {max }}=R_{0}-\frac{3 \ell^{2}}{4 r_{\max }} .
$$

This equation shows that for sufficiently small, but non-zero, $\tilde{t}, r_{\max }<R_{0}$. In the limit $\tilde{t} \rightarrow 0, r_{\max }=R_{0}$. These properties of $r_{\max }$ are consistent with the plots shown in 


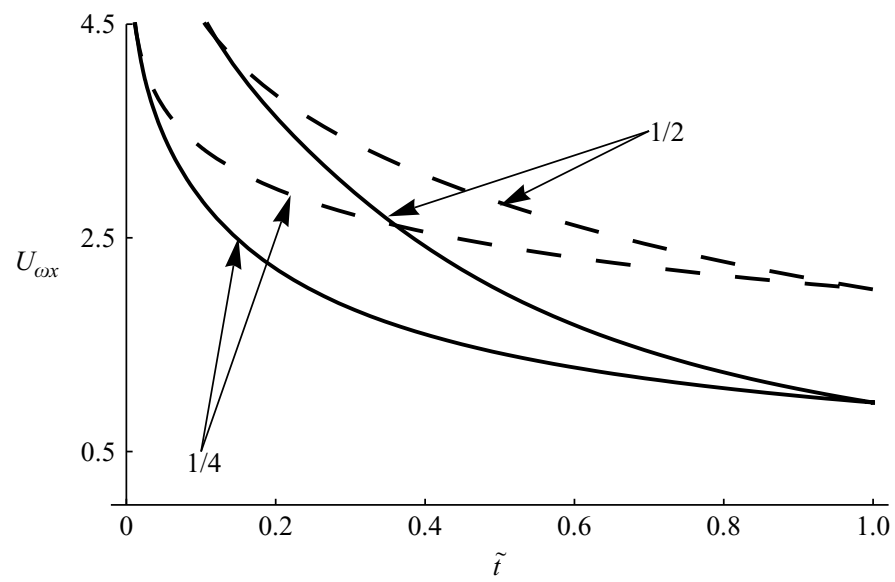

FIGURE 14. The plots of $U_{\omega x}$ versus $\tilde{t}$ as predicted by the general equation (3.16) (solid curves) and the simplified equation (4.22) (dashed curves) for $b=1 / 2$ and $1 / 4$ (numbers near the curves).

figure 7, although the resolution of the curves in the immediate vicinity of $\tilde{t}=0$ is not sufficient to clearly demonstrate the convergence of $r_{\max }$ to $R_{0}$.

Equation (4.23) can be considered a quadratic equation in $r_{\max }$. Its solution in the limit of short times can be presented as

$$
r_{\text {max }}=R_{0}\left(1-\frac{3 \ell^{2}}{4 R_{0}^{2}}\right)=R_{0}\left(1-\frac{3 a^{2} t^{2 b}}{4 R_{0}^{2}}\right) .
$$

Having substituted this equation into (3.17) we obtain

$$
U_{e f f(r)}=-\frac{3 a^{2} b}{2 R_{0} v_{n}} t^{2 b-1} .
$$

As follows from (4.25), for sufficiently small times, $U_{\text {eff }(r)}$ is always negative. This is consistent with our earlier observation that for sufficiently small, but non-zero, $\tilde{t}, r_{\max }<R_{0}$, while in the limit $\tilde{t} \rightarrow 0, r_{\max }=R_{0}$. For $b=1 / 2, U_{\text {eff }(r)}$ remains finite at $\tilde{t} \rightarrow 0$, while for $1 / 4<b<1 / 2, U_{e f f(r)} \rightarrow-\infty$ at $t \rightarrow 0$. These predictions of (4.25) are consistent with the trends of the curves shown in figure 10 for small times.

\section{Theory versus experiments}

The results of experimental studies of vortex rings in various controlled and uncontrolled conditions have been reported in numerous papers (e.g. Shariff \& Leonard 1992; Lim \& Nickels 1995). In the case of classical vortex rings generated in liquids (e.g. water) their basic properties have been explained in terms of the conventional models of laminar rings (Saffman 1970; Saffman 1992; Rott \& Cantwell 1993a,b; Wang et al. 1997; Fukumoto \& Moffatt 2000). In what follows, some of the theoretical results described so far are compared with published experimental data.

The values of $U_{x}$, predicted by (3.14) and (3.25) for $b=1 / 2$, Saffman's formula (1.1) and the upper and lower bounds of the experimental results reported by Weigand \& Gharib (1997) are compared in figure 15. As shown by Weigand \& Gharib (1997), their experimental data in the range of Reynolds numbers between 830 and 1650 lie between the lower and upper boundary curves described by (3.23) with $(k=14.5$; $\left.k^{\prime}=10.6\right)$ and $\left(k=13.6 ; k^{\prime}=7.5\right)$ respectively. The best curve fit for experimental 


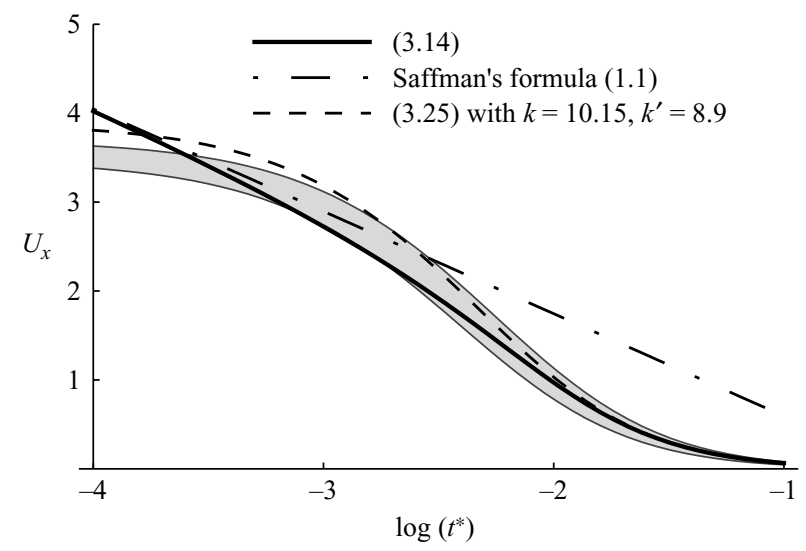

FIGURE 15. The plots of $U_{x}$ versus $\log t^{*}=\log \left[1 /\left(32 \theta^{2}\right)\right]$ based on (3.14) (thick solid curve), (3.25) (dashed curve) for $k=10.153200$ and $k^{\prime}=8.9090909$ and (1.1) (dashed-dotted curve); lower and upper bounds for experimental results by Weigand \& Gharib (1997) correspond to the lower and upper boundaries of the shaded area.

data was achieved for $\left(k=14.4 ; k^{\prime}=7.8\right)$. As can be seen from figure 15 , both plots predicted by (3.14) and (3.25) for $b=1 / 2$ are reasonably close to the experimental results by Weigand $\&$ Gharib (1997) in the range $830 \leqslant R e \leqslant 1650$. This was expected, as the results presented by Weigand \& Gharib (1997) refer to the laminar case. The result predicted by (1.1) do not depend on $a$ or $b$. At $t^{*}>0.01$ the plots based on (3.14) and (3.25) are approximately coincident in agreement with figure 11.

The experimental data obtained by Weigand \& Gharib (1997) refer to real-life vortex rings, produced in the laboratory. At the initial time, these rings did not have delta-function-like structures of the vorticity distribution, which was assumed in solution (3.1). Hence, a noticeable deviation of the experimental plots from the predictions of the model is observed at short times. Note that there is no contradiction between this result and table 1 of Weigand \& Gharib (1997), predicting an almost linear increase in the vortex ring translational velocity with increasing $R e$. This is related to the fact that the velocity in table 1 of Weigand \& Gharib (1997) is dimensional, while the velocity shown in figure 15 is dimensionless and proportional to $V_{x} / \Gamma_{0} \sim V_{x} / \mathrm{Re}$.

Also, an attempt was made to compare the velocities predicted by (3.14) with experimental data reported by Dabiri \& Gharib (2004), who performed experimental studies of isolated vortex rings in water in the range of Re between 2000 and 4000 based on the initial circulations (when vortex rings were first observed). As in the previously described experiments by Weigand \& Gharib (1997), the vortex rings were generated by a piston motion, and they were observed to start approximately $2 \mathrm{~s}$ after the piston was first set to motion. Two values of the ratio of $L$ (stroke) to $D$ (diameter) were considered: 4 and 2 . In the case of $L / D=4$, the observed velocities of the vortex rings were approximated as

$$
V_{x}=5 t^{-0.34},
$$

where $V_{x}$ is in $\mathrm{cm} \mathrm{s}^{-1}$ and $t$ is in s. Normalizing $V_{x}$ by $V_{x}\left(t_{\text {init }}=1 \mathrm{~s}\right)$ and $t$ by $t_{\text {init }}=1 \mathrm{~s}$, (5.1) can be rewritten as

$$
\bar{U}_{x}=\frac{U_{x}}{U_{x}\left(t_{\text {init }}\right)}=\frac{V_{x}}{V_{x}\left(t_{\text {init }}\right)}=\left(\frac{t}{t_{\text {init }}}\right)^{-0.34}=(\bar{t})^{-0.34} .
$$




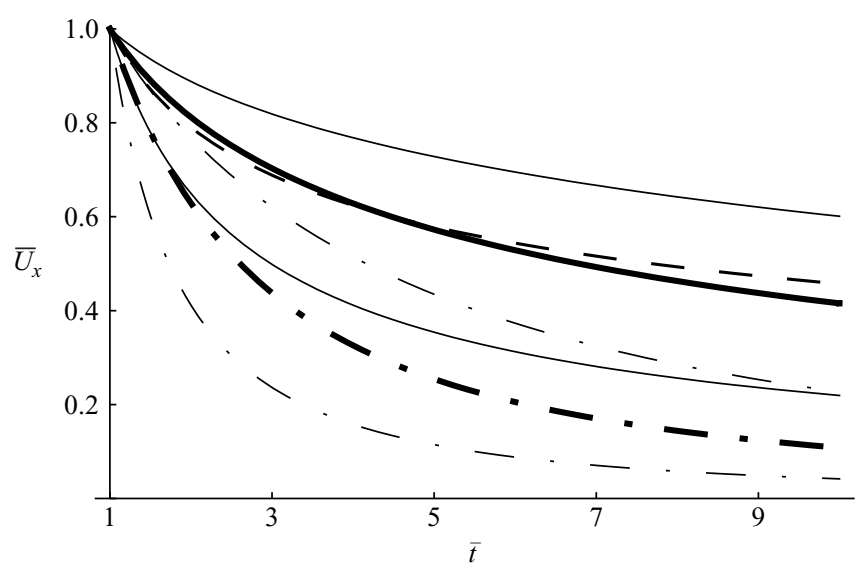

FIGURE 16. The plots of $\bar{U}_{x}=U_{x}(t) / U_{x}\left(t=t_{\text {init }}=1 \mathrm{~s}\right)$ versus $\bar{t}=t / t_{\text {init }}$ as predicted by experimental results by Dabiri \& Gharib (2004) for $L / D=4: \bar{U}_{x}=(\bar{t})^{-0.34}$ (dashed curve) and the model (3.14) for $b=1 / 4$ (solid curves) and $b=1 / 2$ (dashed-dotted curves). Thin solid and dashed-dotted curves refer to $\theta_{\text {init }}=1$ (upper curves) and $\theta_{\text {init }}=4$ (lower curves). Thick solid and dashed-dotted curves refer to $\theta_{\text {init }}=2.5$.

The values of $\bar{U}_{x}$ versus $\bar{t}$ predicted by (5.2) are shown in figure 16 as a dashed curve.

To compare the prediction of (5.2) with (3.14) the values of $\theta_{\text {init }}=\theta\left(t_{\text {init }}\right)$ and $b$ need to be specified. As follows from the analysis by Kaplanski \& Rudi (2005), the values of $\theta_{\text {init }}$ predicted by the slug-flow model are controlled by $L / D$. As follows from figure 2 of Kaplanski \& Rudi (2005), for $L / D=4, \theta_{\text {init }}$ is expected to be in the range from 4 to 1 (the thickest vortex ring, the shape of which can be clearly identified). The model, described earlier in this section, is valid for $1 / 4 \leqslant b \leqslant 1 / 2$. The plots of $\bar{U}_{x}$ versus $\bar{t}$, predicted by (3.14), for $b=1 / 4,1 / 2$ and $\theta_{\text {init }}=1,4$ and 2.5 (averaged between 1 and 4 ), are shown in figure 16. As follows from this figure, in the case of $b=1 / 4$ the predicted values of $\bar{U}_{x}$ are the closest to the experimentally observed values of $\bar{U}_{x}$ when $\theta_{\text {init }}=2.5$. When $\theta_{\text {init }}=1$ the observed values of $\bar{U}_{x}$ are expected to match the predicted ones for $b$ between $1 / 4$ and $1 / 2$. There is no match between the experimentally observed and the predicted values of $\bar{U}_{x}$ for $\theta_{\text {init }}=4$. The case for $L / D=4$ is particularly important for our comparison, as in this case the momentum of vorticity of the observed vortex rings was conserved in the experiment described by Dabiri \& Gharib (2004). The derivation of (3.14) was essentially based upon the assumption that this momentum was conserved.

In the case of $L / D=2$, the values of $\theta_{\text {init }}$ are expected to be in the range of 1 and 26 with the average value equal to 13.5. The agreement between theoretical and experimental results turned out to be the best for $b=1 / 4$ and $\theta_{\text {init }}=13.5$ (the plots are not shown).

In figure 17 the results predicted by (5.2), (3.14) and (3.25) are compared for $\theta_{\text {init }}=2.5$. The results predicted by (3.14) are shown for $b=1 / 4$, while the results predicted by (3.25) are shown for $b=1 / 4$ and $b=1 / 2$. As can be seen from this figure, the results predicted by both (3.14) and (3.25) for $b=1 / 4$ are reasonably close to the results predicted by (5.2). At the same time the results predicted by (3.25) for $b=1 / 2$ are noticeably different from those predicted by (5.2), in agreement with figure 16 . 


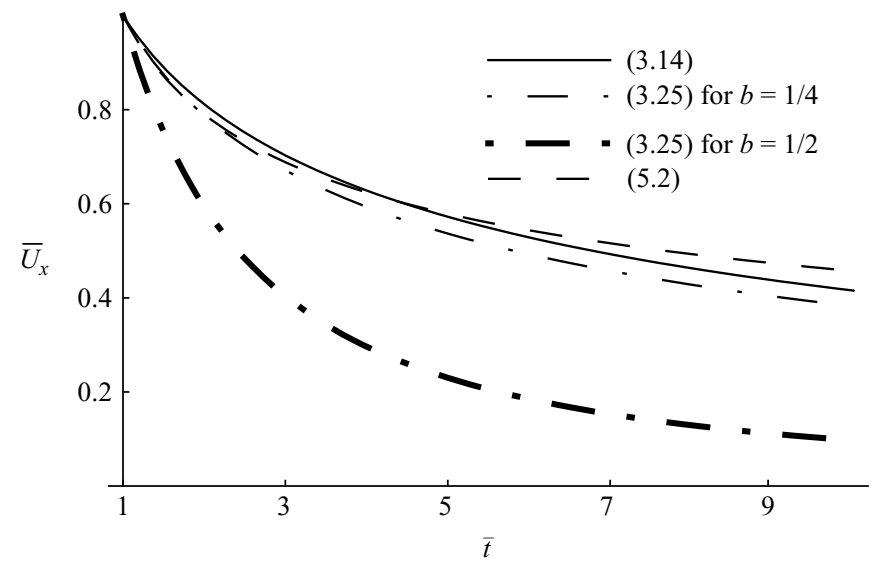

FIGURE 17. The plots of $\bar{U}_{x}=U_{x}(t) / U_{x}\left(t=t_{\text {init }}=1 \mathrm{~s}\right)$ versus $\bar{t}=t / t_{\text {init }}$ as predicted by experimental results by Dabiri \& Gharib (2004) for $L / D=4: \bar{U}_{x}=(\bar{t})^{-0.34}(5.2)$ and the model ((3.15) for $b=1 / 4$ and (3.26) for $b=1 / 4$ and $b=1 / 2)$. All theoretical curves refer to $\theta_{\text {init }}=2.5$.

Note that the experimental results predicted by Maxworthy (1972) in the same range of $R e$ were approximated as (see Dabiri \& Gharib 2004)

$$
\bar{U}_{x}=(\bar{t})^{-1} .
$$

The reliability of these results was questioned by Dabiri \& Gharib (2004). They were not used in our analysis.

\section{Conclusions}

A conventional laminar vortex ring model has been generalized by assuming that the time dependence of the vortex ring thickness $\ell$ is given by the relation $\ell=a t^{b}$, where $a$ is a positive number and $1 / 4 \leqslant b \leqslant 1 / 2$. In the case in which $a=\sqrt{2 v}$, where $v$ is the laminar kinematic viscosity, and $b=1 / 2$, the predictions of the generalized model are identical with the predictions of the conventional model. The time-dependent effective viscosity $v_{*}$ is presented as $\ell \ell^{\prime}$. In the case in which $a=\sqrt{2 v}$ and $b=1 / 2, v_{*}=v$. This generalization was performed in the case of both fixed vortex ring radius $R_{0}$ and increasing vortex ring radius. In the latter case, the socalled second Saffman's formula (see Saffman 1970) has been generalized. The general solutions for vortex ring vorticity, stream function, energy and velocities have been shown to reduce to the previously reported solutions in the cases of long and short times. It has been shown that both vortex ring energy and translational velocity depend strongly on the value of the parameter $b$.

The time evolutions of the locations of the region of maximal vorticity and the region in which the velocity of fluid is equal to zero, in the frame of reference moving with the vortex ring centroid, are found. It is pointed out that the location of both regions depends on $b$, the second region being always further away from the vortex axis than the first one. It is shown that the axial velocities of the fluid in the first region are always larger than the axial velocities in the second region. Both velocities depend strongly on $b$. Although the radial component of velocity in both these regions is equal to zero, the location of both these regions changes with time. This leads to the effective radial velocity component, and the latter depends on $b$. 
Theoretical results have been validated against experimental data reported by Weigand \& Gharib (1997) and Dabiri \& Gharib (2004) in a wide range of Reynolds numbers (based on local circulation).

The authors are grateful to the EPSRC (grant EP/E047912/1) (UK) and the Estonian Science Foundation (grant ETF 6832) for their financial support of this project.

\section{REFERENCES}

Afanasyev, Y. D. \& Korabel, V. N. 2004 Starting vortex dipoles in a viscous fluid: asymptotic theory, numerical simulation, and laboratory experiments. Phys. of Fluids 16 (11), 3850-3858.

Batchelor, G. K. (1967) An Introduction to Fluid Dynamics. Cambridge University Press.

Berezovski, A. \& KaPlansKi, F. 1995 Vorticity distributions for thick and thin vortex pairs and rings. Arch. Mech. 47 (6), 1015-1026.

CAntwell, B. 2002 Introduction to Symmetry Analysis. Cambridge University Press.

DABIRI, J. O. \& GHARIB, M. 2004 Fluid entrainment by isolated vortex rings. J. Fluid Mech. 511, $311-331$

Fukumoto, Y. \& Kaplanski, F. 2008 Global time evolution of an axisymmetric vortex ring at low Reynolds numbers. Phys. of Fluids 20, 053103.

Fukumoto, Y. \& Moffatt, H. K. 2000 Motion and expansion of a viscous vortex ring. Part 1. A higher-order asymptotic formula for the velocity. J. Fluid Mech. 417, 1-45.

Glezer, A. \& Coles, D. 1990 An experimental study of a turbulent vortex ring J. Fluid Mech. 211, 243-283.

Helmholtz, H. 1858 On integrals of the hydrodynamical equations which express vortex-motion. Crelle's J. 55, 485-512.

Kaltaev, A. 1982 Investigation of dynamic characteristics of a vortex ring of viscous fluid (in Russian). In Continuum Dynamics, pp. 63-70. Kazan State University.

Kambe, T. \& Oshima, Y. 1975 Generation and decay of viscous vortex rings J. Phys. Soc. Jpn 38, 271-280.

Kaplanski, F. \& Rudi, U. 1999 Dynamics of a viscous vortex ring. Intl J. Fluid Mech. Res. 26, 618-630.

KaPLANSKI, F. \& RUDI, Y. 2005 A model for the formation of 'optimal' vortex rings taking into account viscosity. Phys. Fluids 17, 087101-087107.

Kovasznay, L. S. G., Fujita, H. \& Lee, R. L. 1974 Unsteady turbulent puffs. Adv. Geophys. 18B, 253-263.

LAMB, H. 1932 Hydrodynamics, 6th edition. Dover.

Lavrentiev, M. A. \& Shabat, B. V. 1973 Problems of Hydrodynamics and Mathematical Models. Nauka.

Lim, T. \& Nickels, T. 1995 Vortex rings. In Fluid Vortices (ed. S. I. Green). Kluwer.

Linden, P. E. \& TuRnER, J. S. 2001 The formation of 'optimal' vortex rings, and the efficiency of propulsion devices. J. Fluid Mech. 427, 61-72.

Lugovtsov, B. A. 1970 On the motion of a turbulent vortex ring and its role in the transport of passive contaminant. In Some Problems of Mathematics and Mechanics. Nauka.

Lugovtsov, B. A. 1976 On the motion of a turbulent vortex ring. Arch. Mech. 28, 759-766.

Mathematica 2007 Version 6.0.0. http://functions.wolfram.com.

Maxworthy, T. 1972 The structure and stability of virtex rings. J. Fluid Mech. 51, 15-32.

MAXwORTHY, T. 1974 Turbulent vortex rings (with an appendix on an extended theory of laminar vortex rings). J. Fluid Mech. 64, 227-239.

Maxworthy, T. 1977 Some experimental studies of vortex rings. J. Fluid Mech. 81, 465-495.

MoHSENI, K. 2001 Statistical equilibrium theory of axisymmetric flows: Kelvin's variational principle and an explanation for the vortex ring pinch-off process. Phys. Fluids 13, 1924-1931.

MoHSENI, K. 2006 A formulation for calculating the translational velocity of a vortex ring or pair. Bioinspir. Biomim. 1, S57-S64. 
Mohseni, K. \& Gharib, M. 1998 A model for universal time scale of vortex ring formation. Phys. Fluids 10, 2436-2438.

Norbury, J. 1973 A family of steady vortex rings. J. Fluid Mech. 57, 417-431.

Panton, R. L. 1996 Incompressible Flow. John Wiley.

Phillips, O. M. 1956 The final period of decay of non-homogeneous turbulence. Proc. Camb. Phil. Soc. 252, 135-151.

Rott, N. \& Cantwell, B. 1993 a Vortex drift. Part 1. Dynamic interpretation Phys. Fluids A 5, $1443-1450$.

Rott, N. \& Cantwell, B. $1993 b$ Vortex drift. Part 2.. The flow potential surrounding a drifting vortical region. Phys. Fluids A 5, 1451-1455.

Saffman, P. G. 1970 The velocity of viscous vortex rings. Stud. Appl. Math. 49, 371-380.

Saffman, P. G. 1992 Vortex Dynamics. Cambridge University Press.

Sazhin, S. S., Kaplanski, F., Feng, G., Heikal, M. R. \& Bowen, P. J. 2001 A fuel spray induced vortex ring. Fuel 80, 1871-1883.

Shariff, K. \& LeOnard, A. 1992 Vortex rings. Annu. Rev. Fluid Mech. 24, 235-279.

Shusser, M. \& Gharib, M. 2000 Energy and velocity of a forming vortex ring. Phys. Fluids 12, 618-621.

Stanaway, S., Cantwell, B. J. \& Spalart, P. R. 1988 A numerical study of viscous vortex rings using a spectral method. Tech. Memo. 101041. NASA.

Wang, C.-T, Chu, C.-C. \& Chien-Chang 1994 Initial motion of a vortex ring. Proc. R. Soc. London A 446, 589-599.

Weigand, A. \& Gharib, M. 1997 On the evolution of laminar vortex rings. Exps Fluids 22, 447-457. 